\title{
Title: Mesenchymal stromal cell remodeling of a gelatin hydrogel microenvironment defines an artificial hematopoietic stem cell niche
}

\author{
Authors: Aidan E. Gilchrist ${ }^{1}$, Sunho Lee ${ }^{2}$, Yuhang $\mathrm{Hu}^{2}$, Brendan A.C. Harley ${ }^{3,4, *}$ \\ Affiliations: \\ ${ }^{1}$ Dept. of Materials Science and Engineering, University of Illinois at Urbana-Champaign \\ Urbana, IL 61801 \\ ${ }^{2}$ Dept. of Mechanical Science and Engineering, University of Illinois at Urbana-Champaign \\ Urbana, IL 61801 \\ ${ }^{3}$ Dept. of Chemical and Biomolecular Engineering, University of Illinois at Urbana-Champaign \\ Urbana, IL 61801 \\ ${ }^{4}$ Carl R. Woese Institute for Genomic Biology, University of Illinois at Urbana-Champaign \\ Urbana, IL 61801
}

*To whom correspondence should be addressed:

B.A.C. Harley

Dept. of Chemical and Biomolecular Engineering

Carl R. Woese Institute for Genomic Biology

University of Illinois at Urbana-Champaign

110 Roger Adams Laboratory

600 S. Mathews Ave.

Urbana, IL 61801

Phone: (217) 244-7112

Fax: (217) 333-5052.

One Sentence Summary: Coupling effects of hydrogel biotransport, heterotypic cell culture, and matrix remodeling enhances hematopoietic stem cell culture and quiescence.

\footnotetext{
Abstract: Hematopoietic stem cells (HSCs) reside in the bone marrow within discrete niches defined by a complex milieu of external signals including biophysical cues, bound and diffusible biomolecules, and heterotypic cell-cell interactions. Recent studies have shown the importance of autocrine-mediated feedback of cell-secreted signals and the interplay between matrix architecture and biochemical diffusion on hematopoietic stem cell activity. Autocrine and paracrine signaling from HSCs and niche-associated mesenchymal stromal cells (MSCs) have both been suggested to support HSC maintenance in vivo and in vitro. Here we report the
} 
development of a library of methacrylamide-functionalized gelatin (GelMA) hydrogels to explore the balance between autocrine feedback and paracrine signals from co-encapsulated murine bone marrow MSCs on murine HSCs. The use of a degradable GelMA hydrogel enables the possibility for significant MSC-mediated remodeling, yielding dynamic shifts in the matrix environment surrounding HSCs. We identify a combination of an initially low-diffusivity hydrogel and a 1:1 HSPC:MSC seeding ratio as conducive to enhanced HSC population maintenance and quiescence. Further, gene expression and serial mechanical testing data suggests that MSC-mediated matrix remodeling is significant for the long-term HSC culture, reducing HSC autocrine feedback and potentially enhancing MSC-mediated signaling over 7-day culture in vitro. This work demonstrates the design of an HSC culture system that couples initial hydrogel properties, MSC co-culture, and concepts of dynamic reciprocity mediated by MSC remodeling to achieve enhanced HSC maintenance.

\section{[Main Text: ]}

\section{Introduction}

Hematopoietic stem cells (HSCs) possess the capacity for self-renewal and can repopulate the body's entire complement of blood and immune cells through a highly regulated differentiation hierarchy (1-3). Following development, HSCs lodge primarily in the bone marrow in unique tissue microenvironments termed niches, which provide a milieu of extrinsic signals in the form of biophysical cues, biomolecular gradients, and co-inhabiting niche cells that together promote quiescence, self-renewal, and lineage specification (4-9). Any disruptions to the niche can lead to a loss regulation and abnormal or unsuccessful hemostasis in the bone marrow. The resulting drop in HSC efficacy from niche disruption has hindered clinical use of HSC transplants (HSCTs) for the treatment of blood and immune disorders $(10,11)$. There is an 
acute clinical need for in vitro approaches to improve HSC expansion or preconditioning to facilitate improved homing and engraftment of transplanted donor HSCs to recipient bone marrow niches. Such investigations require biomaterial culture systems that can provide the correct sequence of signals to enhance HSC expansion in vitro. While exact replication of the native niche is prohibitively complex due to its transient nature, heterogeneity, and coupling of external factors, insight from the native niche can inform the development of a synthetic culture platform that mediates HSC response (12).

Knockout (depletion) and proximity principal (localization) models, both in vivo and in vitro, have been used to elucidate a range of triggers present in the microenvironment that maintain HSC hematopoietic activity (13). In vivo, HSCs are presented with complex patterns of ligands such as laminins and fibronectin $(14,15)$ and an effective Young's modulus ranging from 0.25 to $24.7 \mathrm{kPa}$ (16). Matrix biophysical cues within the bone marrow such as elasticity and substrate stiffness can also significantly influence HSC expansion and migration (17-20). More recently, tissue engineering approaches have begun to identify critical biomaterial design parameters inspired by native niche signals for ex vivo HSC culture. Notably, elastic substrates functionalized with fibronectin have been shown to induce ex vivo expansion of HSC progenitor populations (21). However, in addition to direct effects of the niche microenvironment on HSC activity, it is increasingly important to examine indirect effects as well. For example, the niche microenvironment can modulate the behavior of co-inhabiting niche cells, which can then exert an influence on HSC activity via cell-cell contact (e.g., cadherin interactions) and secreted biomolecular factors $(22,23)$. Further, the ECM can modulate biotransport of secreted factors via transient binding motifs (e.g. proteoglycans) or steric hindrance to diffusive and advective mass 
transport (24-27). Design of artificial niches offers the opportunity to regulate the transport of biomolecular signals in heterotypic cultures, and engineer HSC-generated autocrine feedback and niche cell paracrine signals. Work by Pompe et al. demonstrates this potential with a microcavity system of single vs. doublet HSC cultures, with autocrine feedback dominating quiescent HSC maintenance (28).

Expanding upon recent use of exogenous stimuli (e.g. conditioned media) to mimic cellsignaling motifs $(29,30)$, biomaterial design can exploit matrix properties to create regimes of favored cell-signaling mechanism. In a tight matrix, with a small mesh size and low diffusivity, HSCs may experience a small radius of communication, as cell-cell communication is hindered by steric and electrostatic interactions with the matrix, enforcing a mostly autocrine feedbackrich environment. Alternatively, in a loose matrix, with a large mesh size, HSCs have a larger radius of communication, allowing for possible heterotypic paracrine signaling. Culturing hematopoietic lineage positive cells in a three-dimensional collagen system, we recently demonstrated improved maintenance of early progenitor HSC populations in autocrinedominated environments with low diffusivity (31). While promising, this early-generation hydrogel system was not optimal for in-depth studies of the intersection of heterotypic cell interactions and cell mediated remodeling.

Bone marrow mesenchymal stromal cells (MSCs) show significant promise for the development of artificial bone marrow platforms for ex vivo HSC culture. MSCs co-localize with HSCs in the perivascular regions and central marrow and influence hematopoietic activity (32, 33). Paracrine signaling from proximal MSCs are believed to mediate HSC activation and 
quiescence via direct cell-cell contact and diffusive signaling $(5,28,33-37)$ through production of factors such as CXCL12 (38), IL-6 (3, 39), and TPO (40). Biomaterial systems offer the opportunity to identify heterotypic HSC-MSC cultures that support HSC quiescence or selfrenewal. However, studies of HSC-MSC interactions are significantly complicated by processes of dynamic reciprocity $(41,42)$. Notably, while the matrix can impart cues to directly influence MSC-HSC interactions, MSCs are also equipped to remodel the matrix via deposition of proteins and enzymatically degradation of the matrix $(41,43-45)$, leading to non-static material properties, and a dynamic feedback loop. MSCs can also create strain gradients and deformation in the local environment, the so-called peri-cellular region (44). As a result, it is important to not only consider initial properties of a biomaterial culture system, but to employ methods to interrogate dynamic shifts in these properties, and their effect on resultant domains of signaling. The intricacies of HSC-MSC co-cultures offer unique opportunities to interrogate effects of dynamic reciprocity as a design paradigm in ex vivo HSC culture systems.

The objective of this study was to examine the effect of MSC co-culture on HSC quiescence and differentiation patterns within a three-dimensional methacrylamidefunctionalized gelatin (GelMA) hydrogel. We employ a library of GelMA hydrogels to examine the integrated influence of hydrogel poroelastic properties, paracrine signals, and MSC-mediated matrix remodeling on HSC lineage specification events. Myeloid lineage specification and progenitor cell quiescent events were examined for murine HSCs cultured in GelMA hydrogels in the presence of increasing concentrations of murine bone marrow MSCs (Fig. 1). We also examined HSC differentiation in the context of hydrogel remodeling by MSCs. Together this work represents an integrated approach to consider dynamic biotransport processes as essential 


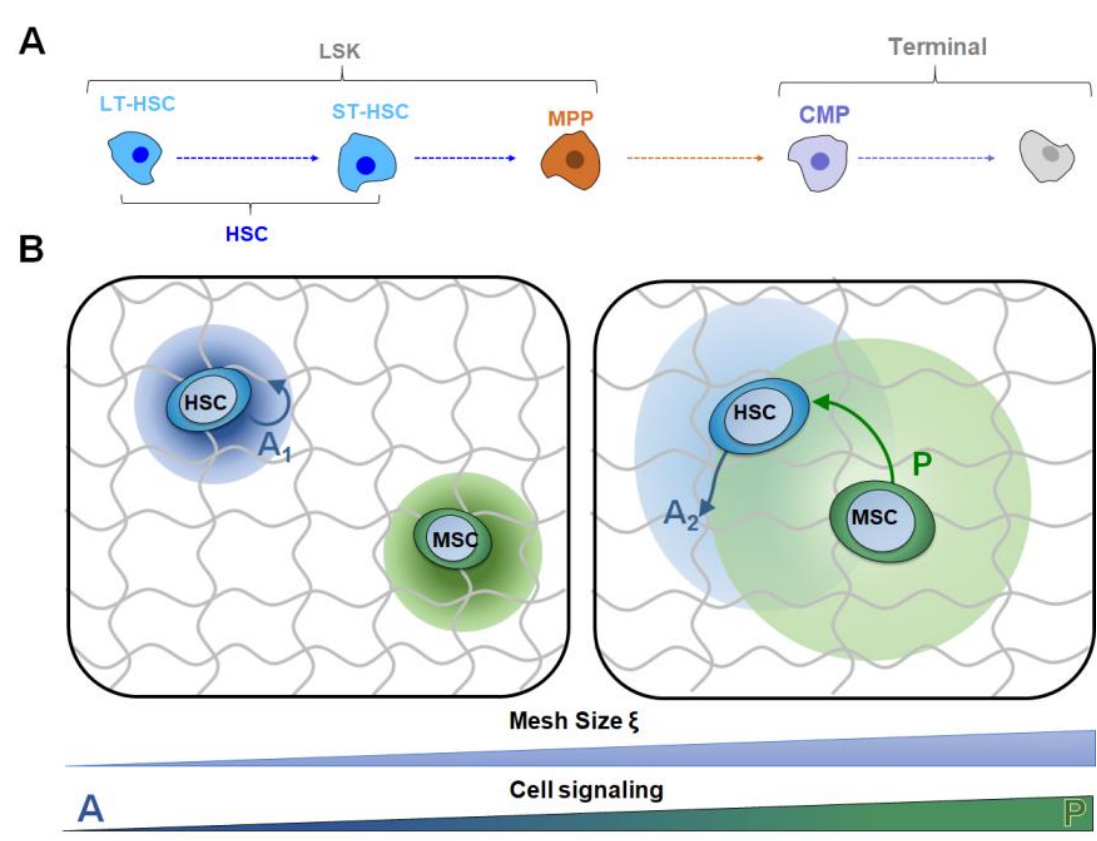

Figure 1. Hematopoietic cell-cell interactions in a dynamic biomaterial landscape. A) Hematopoietic lineage hierarchy. Long-term repopulating HSCs and Short-term repopulating HSCs are grouped as a singular HSC population. HSCs and Multipotent Progenitors comprise the HSC progenitor, HSPCs, population. Common Myeloid Progenitors and Lin+ cells make up the Terminal cell population. B) Autocrine and paracrine signaling can be tuned by adjusting seeding density and altering biotransport properties. In an HSC-only culture, an increase in mesh size leads to a diffusive regime that increases paracrine signaling. In an HSC-MSC co-culture, small mesh constrains secreted biomolecules and leads to a mostly autocrine signaling. However, as mesh size is increased, autocrine feedback is reduced and paracrine signaling begins to dominate cell-cell interactions.

design paradigms in biomaterials for the culture and expansion of primary HSC population.

\section{Results}

\subsection{Poroelastic characterization of GelMA hydrogels}


A library of methacrylamide-functionalized gelatin (GelMA) was assembled and poroelastic parameters were examined in response to changing the degree of methacrylamide functionalization (DOF) of the gelatin macromer, GelMA wt $\%$, and photoinitiator (PI) concentration. The Young's modulus (E) of the library spanned a range of marrow mimetic values $(2.67 \pm 1.39$ to $32.23 \pm 10.7 \mathrm{kPa})$, and showed strong dependence on GelMA wt $\%$ and, to a lesser extent, degree of functionalization (DOF) of the GelMA (Fig. S1). PI (0.05, 0.1\%) was found to only nominally impact the modulus and subsequent analyses here concentrates on the 0.1\%PI condition (Fig. 2A). The subset of hydrogels fabricated from 4wt $\%$ GelMA demonstrated moduli ranging from $4.67 \pm 3.20(35 \% \mathrm{DOF})$ to $7.65 \pm 3.90 \mathrm{kPa}(85 \% \mathrm{DOF})$. A midrange subset (5wt\% GelMA) showed increased elastic moduli range, from $8.90 \pm 1.83$ (35\% DOF) to $13.54 \pm 4.81 \mathrm{kPa}(85 \% \mathrm{DOF})$. The highest GelMA content subset $(7.5 \mathrm{wt} \%)$ demonstrated a range of moduli spanning $17.89 \pm 10.99(35 \% \mathrm{DOF})$ to $30.06 \pm 12.35 \mathrm{kPa}(35 \% \mathrm{DOF})$. Following this analysis, we examined the poroelastic properties of the library of hydrogels in terms of the shear modulus $(G)$ and the diffusion coefficient of water $\left(D_{w}\right)$. Here, the range of shear modulus spanned $0.36 \pm 0.03$ to $6.05 \pm 0.50 \mathrm{kPa}(\mathrm{G})$ while water diffusion ranged from $75.86 \pm 11.84$ to $211.13 \pm 51.84 \mu \mathrm{m}^{2} / \mathrm{s}$ (Fig. S2 and S3).

From this complete library of hydrogels, we identified a subset of three hydrogels (85\% DOF, $0.1 \%$ PI, and 4, 5, 7.5\%GelMA) that spanned a range of mechanical properties associated with the native niche: Low, $\mathrm{E}_{\text {Low }}=4.67 \pm 3.20 ;$ Med, $\mathrm{E}_{\text {Med }}=13.54 \pm 4.81 ;$ High, $\mathrm{E}_{\text {High }}=$ $30.06 \pm 12.35 \mathrm{kPa}$ (Fig. 2A). This subset also displayed a significant increase in the shear modulus $\left(\mathrm{G}_{\text {Low }}=0.51 \pm 0.03 ; \mathrm{G}_{\text {Med }}=1.74 \pm 0.22 ; \mathrm{G}_{H i g h}=4.80 \pm 0.3 \mathrm{kPa}\right.$ respectively $)($ Fig. 2B $)$. Low and Med hydrogels displayed similar water diffusion characteristics $\left(157.19 \pm 24.45,195.04 \pm 24.66 \mu \mathrm{m}^{2} / \mathrm{s}\right)$ 
whereas the High hydrogel variant displayed a significantly reduced $\mathrm{D}_{\mathrm{w}}\left(99.48 \pm 15.11 \mu \mathrm{m}^{2} / \mathrm{s}\right)$

(Fig. 2C). Hydrogel mesh size ( $\xi$ ) calculations showed a mirror inverse of the moduli, with decreased mesh size associated with increased GelMA content: $\xi_{\text {Low }}=72, \xi_{\text {Med }}=58, \xi_{\text {High }}=32 \mathrm{~nm}$ (Fig. 2D).

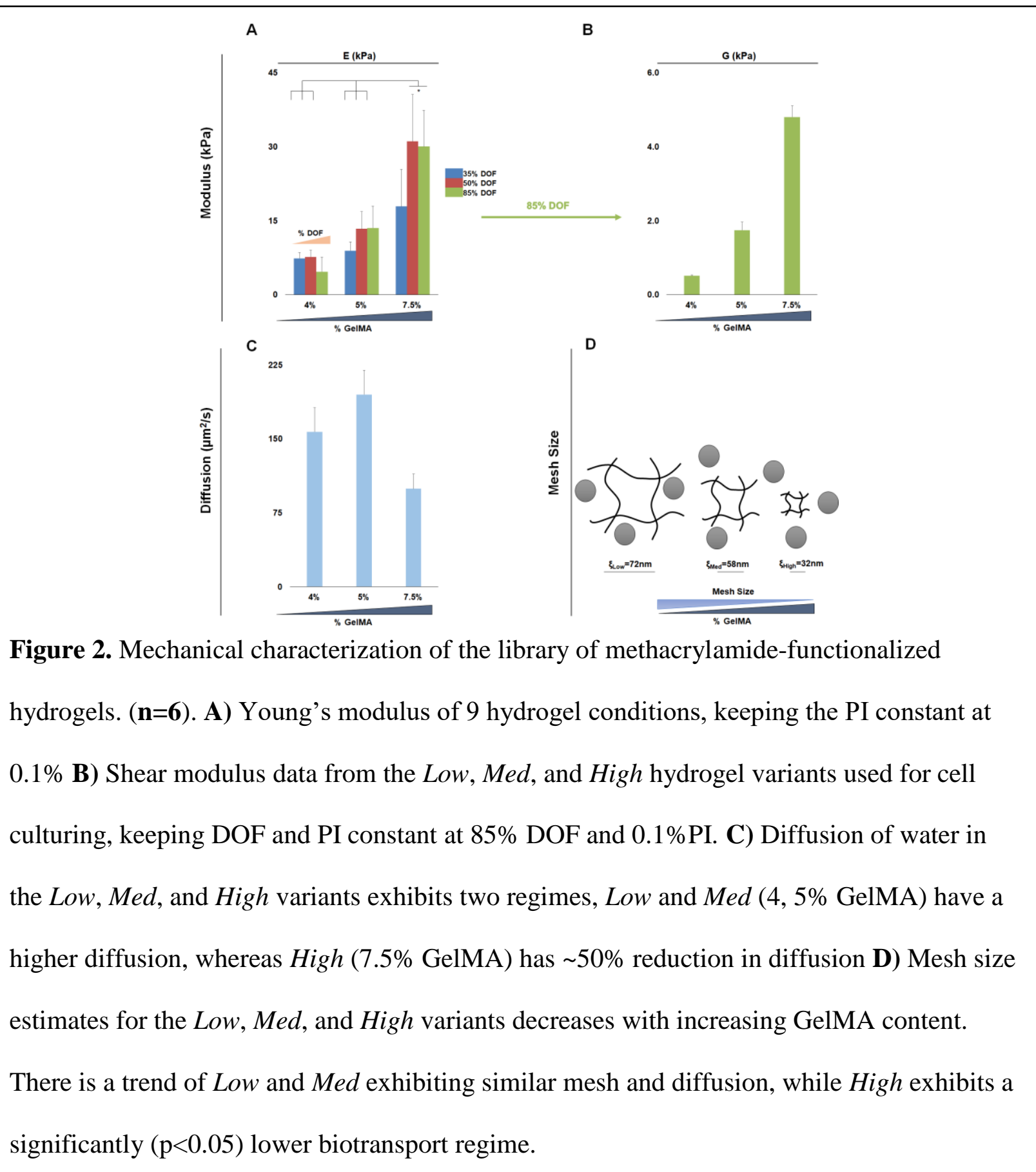




\subsection{MSC mediated remodeling of GelMA hydrogels}

As MSCs can interact and remodel gelatin-based hydrogels, we quantified MSC remodeling of the Low, Med, and High hydrogels via compressive tests as a function of time in culture ( 0 - 7 days) and MSC seeding density $\left(0,1 \times 10^{5}, 1 \times 10^{6}\right.$ cells $\left./ \mathrm{mL}\right)$, with results reported as absolute values as well as normalized against the initial properties (day 0) of each variant (Fig. 3B). Notably, no observable trends in modulus change were observed over time in either Low and Med hydrogel variants. However, the High hydrogel (7.5\%GelMA; $85 \%$ DOF; 0.1\%PI) displayed significant remodeling over time for the highest MSC seeding density $\left(1 \times 10^{6}\right.$ MSCs/mL). Here, significant biosynthetic remodeling was observed, characterized by significant increases in Young's modulus $(\Delta \mathrm{E}=2.61 \pm 0.72)$, almost twice that of other seeding conditions $\left(\Delta \mathrm{E}=1.13 \pm 0.45\right.$, acellular; $\left.\Delta \mathrm{E}=1.25 \pm 0.13,1 \times 10^{5} \mathrm{MSCs} / \mathrm{mL}\right)($ Fig. 3A $)$.

Expression of remodeling-associated genes overtime was examined to determine local changes in remodeling. PCR shows a downregulation of remodeling associated genes in MSCs as early as day 1 , however there were a number of interesting trends across groups. MMP-9 saw the largest upregulation with around 6-fold increase on day 1 across all hydrogel variants in the lowest seeding density (Fig. 4A). The high seeding density, $1 \times 10^{6} \mathrm{MSC} / \mathrm{mL}$ had the largest MMP-9 upregulation, with $10.8 \pm 1.93$ fold increase on day 1 in the Low hydrogel (Fig. 4B). The MMP-activity repressor, tissue inhibitor of metalloprotease, TIMP-1 showed modest upregulation at day 1 (1.3-fold for $1 \times 10^{5} \mathrm{MSCs} / \mathrm{mL}$; 1.5 -fold for $\left.1 \times 10^{6} \mathrm{MSCs} / \mathrm{mL}\right)$ for each hydrogel condition (Low, Med, High), however by intermediate time point, day 4, TIMP-1 had become downregulated. TIMP-2, TIMP-3, and Col1( $\alpha 1)$ were sharply downregulated by day 4 


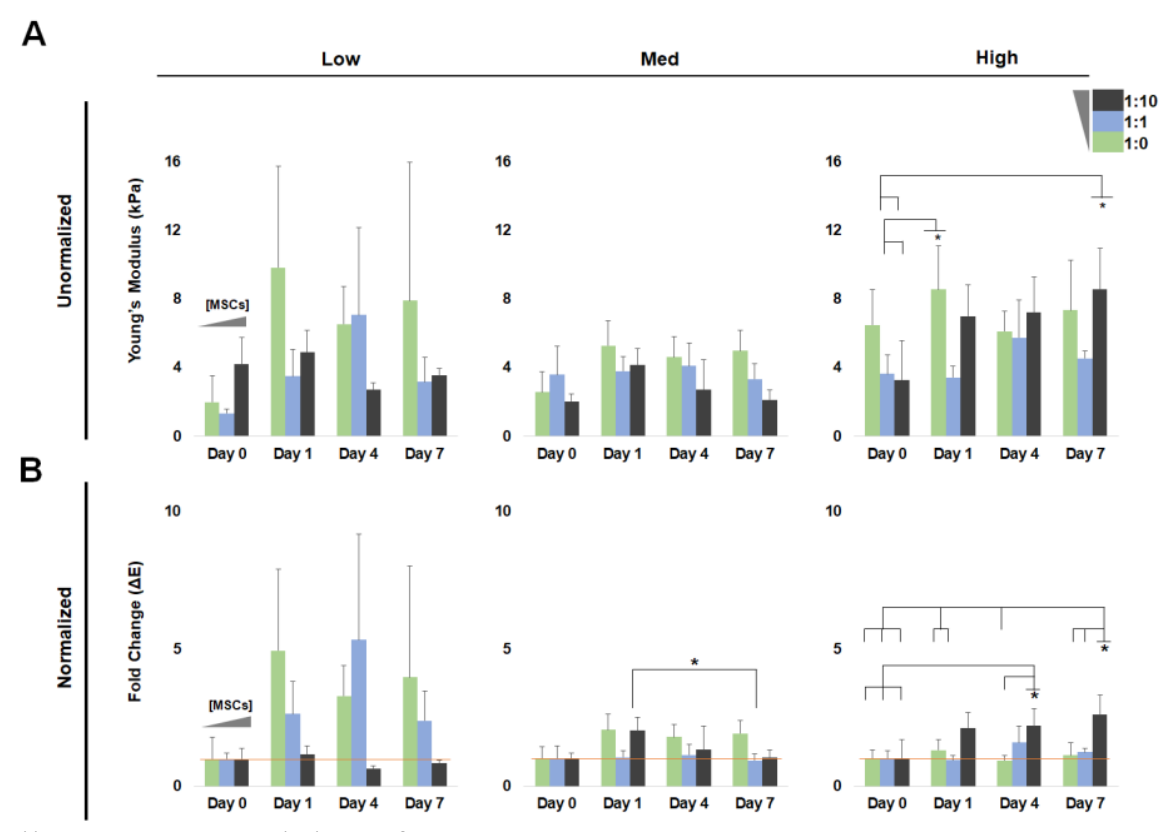

Figure 3. Bulk Young's modulus of Low, Med, and High hydrogels cultured over 7 days with seeding densities of $0,1 \times 10^{5}$, and $1 \times 10^{6} \mathrm{MSCs} / \mathrm{mL}$ (1:0, 1:1, 1:10 HSPCs:MSCs). (n=3-6). A) There is minimal change in Young's modulus over time in any hydrogel and seeding conditions. The high hydrogel shows a significant increase $(\mathrm{p}<0.05)$ from initial seeding to day 7 in the presence of a high concentration of the highest density of MSCs, $1 \times 10^{6}$ MSCs/mL. B) The Young's modulus was normalized to the initial value, day 0, of the specific seeding and hydrogel variant to show fold change. This highlights that only the High with the highest concentration of $\mathrm{MSCs}\left(1 \times 10^{6} \mathrm{MSCs} / \mathrm{mL}\right)$ experiences significant increase $(p<0.05)$ in bulk Young's modulus over time.

while MMP-2 showed decreased expression that increased with increasing MSC density $1 \times 10^{5}$ vs. $\left.1 \times 10^{6} \mathrm{MSCs} / \mathrm{mL}\right)($ Fig. 4A,B)

\subsection{Culture system dependent hematopoietic differentiation patterns}

Murine hematopoietic stem progenitor cells (HSPCs) were cultured in the characterized

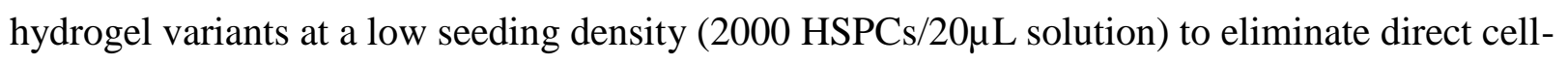


cell contact. Analysis of the homotypic culture displays an HSC population dependence on

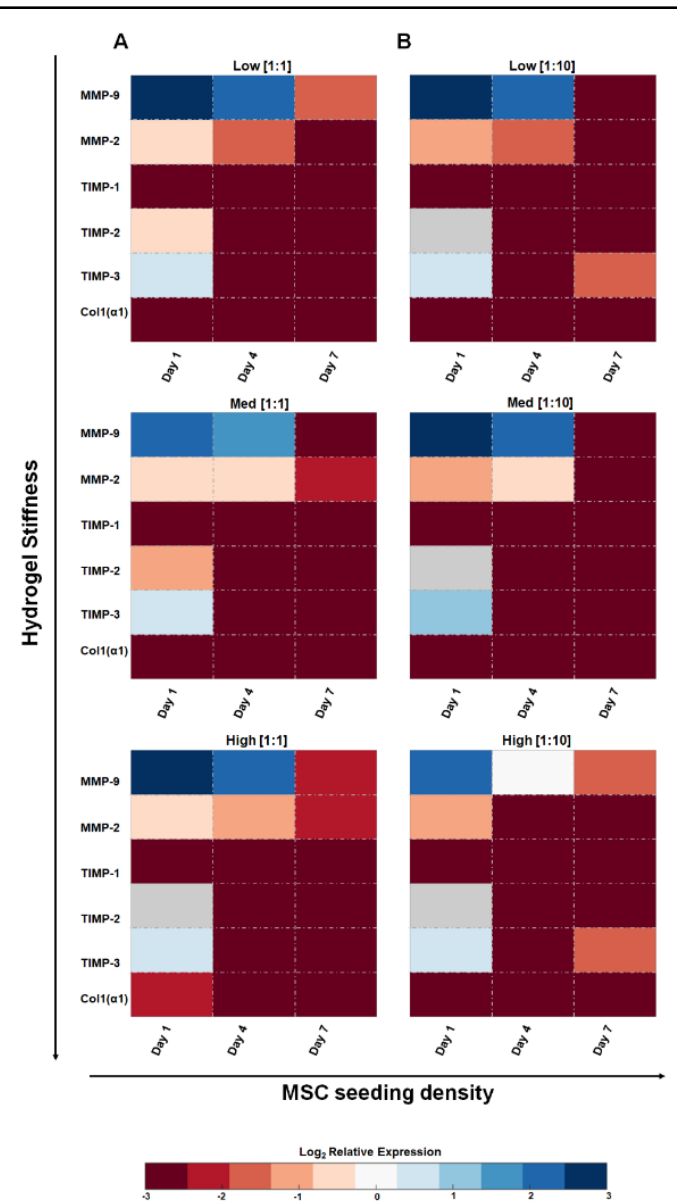

Figure 4. Relative gene expression of matrix-associated genes normalized to day 0 expression, shown on a $\log 2$ scale, with downregulation shown in red and upregulation shown in blue. Gray values for TIMP-2 show non-determinant values, indicative of low yields of expression. Matrix degradation genes analyzed were matrix metalloproteases MMP-2 and MMP-9. Matrix additive genes were the tissue inhibitors of metalloproteases TIMP-1, TIMP2, and TIMP-3, along with the matrix protein collagen, type 1 , alpha $1 .(\mathbf{n}=3-6)$. A) Gene expression of hydrogels seeded with low density of MSCs $1 \times 10^{5} \mathrm{MSCs} / \mathrm{mL}(1: 1$

HSPCs:MSCs). B) Gene expression of hydrogels seeded with high density of MSCs $1 \times 10^{6}$ MSCs/mL (1:10 HSPCs:MSCs).

hydrogel condition: Low, Med, and High (Fig. 5B,C). The percentage of HSCs followed an 
inverse trend, decreasing with increasing GelMA concentration (Fig. 5C). Not unexpectedly, with the decrease in early progenitors, there was an associated increase in the downstream cell population patterns. This was seen in the Common Myeloid Progenitors (CMP) cell population which made up $4.95 \pm 1.02 \%$ of the hematopoietic cell population in the Low hydrogel, but was significantly larger in the High hydrogel, $13.72 \pm 2.93 \%$ (Fig. 5B). A similar trend was seen in the terminal cell population, in which increasing GelMA content led to increasing hematopoietic

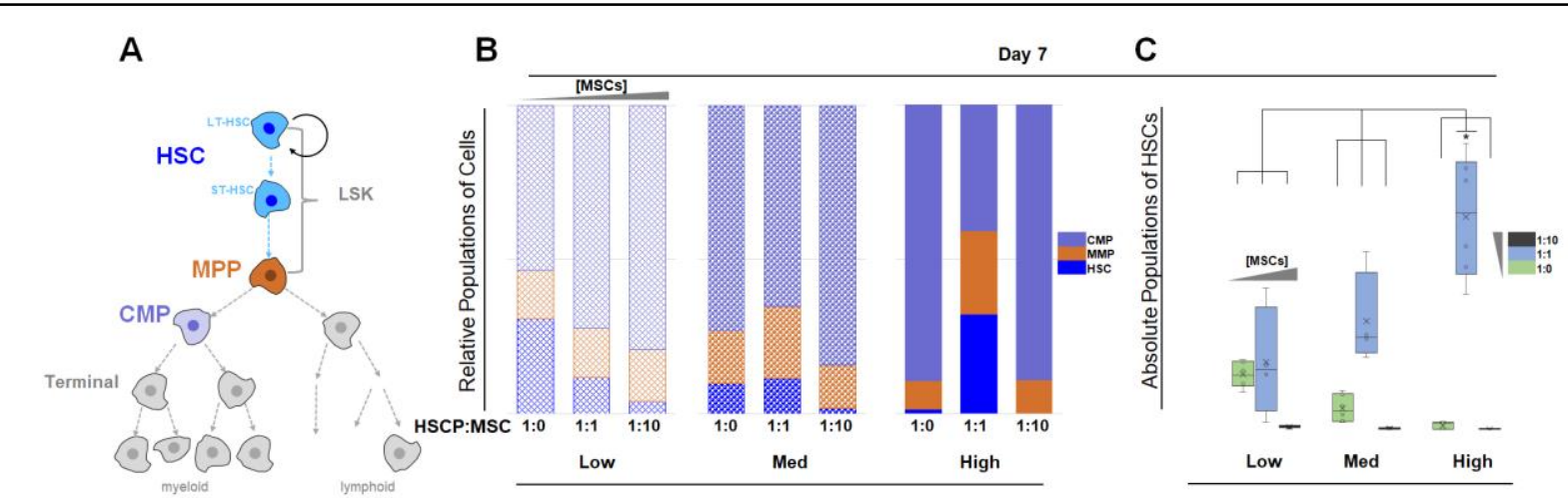

Figure 5. Analysis of cell populations at day $7(\mathbf{n = 6})$. A) The lineage hierarchy of hematopoietic stem cells, with long-term and short-term hematopoietic stem cells grouped as HSCs (blue), Multipotent Progenitors (orange), and Common Myeloid Progenitors (lavender).

B) The relative population of HSC (blue), MPP (orange), CMP (lavender) are shown for single-culture and co-culture at 1:1 and 1:10 (HSPCs:MSCs), in the Low, Med, and High variants. The height of each subcolumn reflects how much of the total cell population is made up of the specified cell type. The blue columns (HSC) show that the highest percentage of HSCs are found in the single-culture Low variant (1:0), and in the 1:1 co-culture High variant. C) The percentage of HSCs that make up the total hematopoietic cell population, arranged in order of increasing MSC density (1:0, 1:1, 1:10 HSPCs:MSCs), and in order of increasing hydrogel stiffness (Low, Med, and High). The largest percent of HSC is found in the High variant at a 1:1 seeding ratio and is significantly higher $(\mathrm{p}<0.05)$ than all other conditions. 
terminal cell population (Fig. S6).

Murine MSCs were also co-cultured with HSPCs in heterotypic cultures with seeding densities kept low to reduce cell-cell contact and ensure only soluble biochemical interactions between cells. These co-culture systems led to important shifts in HSC differentiation patterns (Fig. 5). Where in HSC single-cultures the highest final percentage of early progenitor cells was found in the lower GelMA hydrogels, the addition of MSCs in a 1:1 [HSPCs:MSCs] ratio created a reversal in that trend. The highest maintenance was found in the High hydrogel variant with HSCs comprising $11.11 \pm 3.18 \%$ of the hematopoietic population, while the fraction of remaining HSCs was significantly lower in the Med and Low variants (Fig. 5B,C). From day 1 to day 7 this represents maintenance of 50.2\%, 22.08\%, and $18.50 \%$ of the initial HSPC seeding for High, Med, and Low (Fig. 6). Higher HSC maintenance led to a lower terminal cell population of $77.54 \pm 5.83 \%$ for High hydrogel variants (compared to $82.70 \pm 6.09 \%$ and $91.50 \pm 4.67 \%$ for $\mathrm{Med}$ and Low hydrogel variants; Fig. S6). When HSPCs were co-cultured with a higher density of MSCs ( $\left.1 \times 10^{6} \mathrm{MSCs} / \mathrm{mL}\right)$, at a ratio of 1:10 [HSPCs:MSC], hematopoietic differentiation towards late-stage lineage was enhanced (Fig. 5B,C). Not surprisingly, terminal cells made up the bulk of the hematopoietic population in the 1:10 cultures, representing more than $99 \%$ of the hematopoietic cells across all variants (Fig. S6).

\subsection{Culture dependent shifts in HSC quiescence}

We subsequently performed cell cycle analysis for a subset of culture conditions that led to the greatest maintenance of an HSC population when cultured in the absence (Low 1:0) vs. presence 


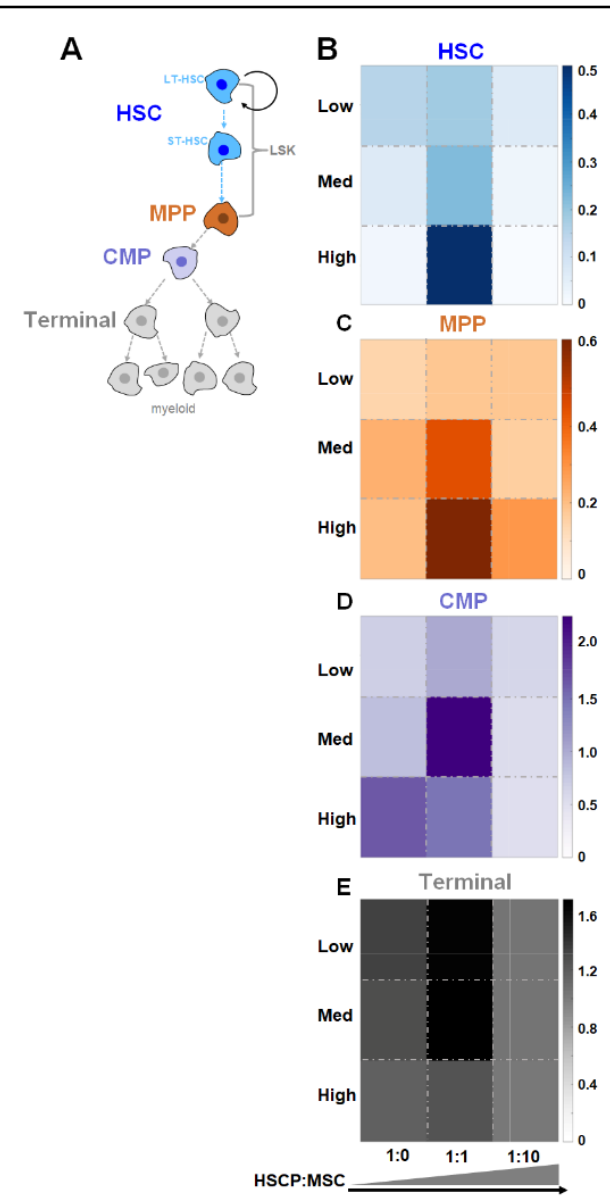

Figure 6. Relative fold change of cell population from day 1 to day 7. A) Color-coded hematopoietic lineage hierarchy B) Fold change of HSC population. Darker blue indicates higher maintenance of HSC (highest maintenance in High variant at 1:1 seeding). C) Fold change of MPP population. Darker orange indicates higher maintenance of MPP (highest in High variant at 1:1 seeding). D) Fold change of CMP population. Darker purple indicates higher maintenance of CMP (highest in Med variant at 1:1 seeding). E) Fold change of Terminal population. Darker gray indicates higher maintenance of Terminal population (highest in Med variant at 1:1 seeding).

(High 1:1) of MSCs. Here we compare the effect of inclusion of MSCs within the hydrogel environment on maintenance of quiescent HSCs, a key parameter for long-term culture success. Across each cell population and data representation, the inclusion of MSCs in a low diffusive 
environment (High 1:1) led to an increase in the number of quiescent (G0-state) hematopoietic cells. Notably, the inclusion of MSCs lead to a 129-fold (LT-HSC subpopulation) and a 7.7-fold (entire HSC population) increase in the number of quiescent cells versus monocultures of HSCs alone in a highly diffusive environment (Low 1:0) (Fig. 7). Further, we observed enhanced HSC quiescence as a result of the presence of the hydrogel matrix compared to conventional liquid cultures of HSCs alone or equivalent HSC-MSC mixtures (Fig. S7).

\section{Discussion}

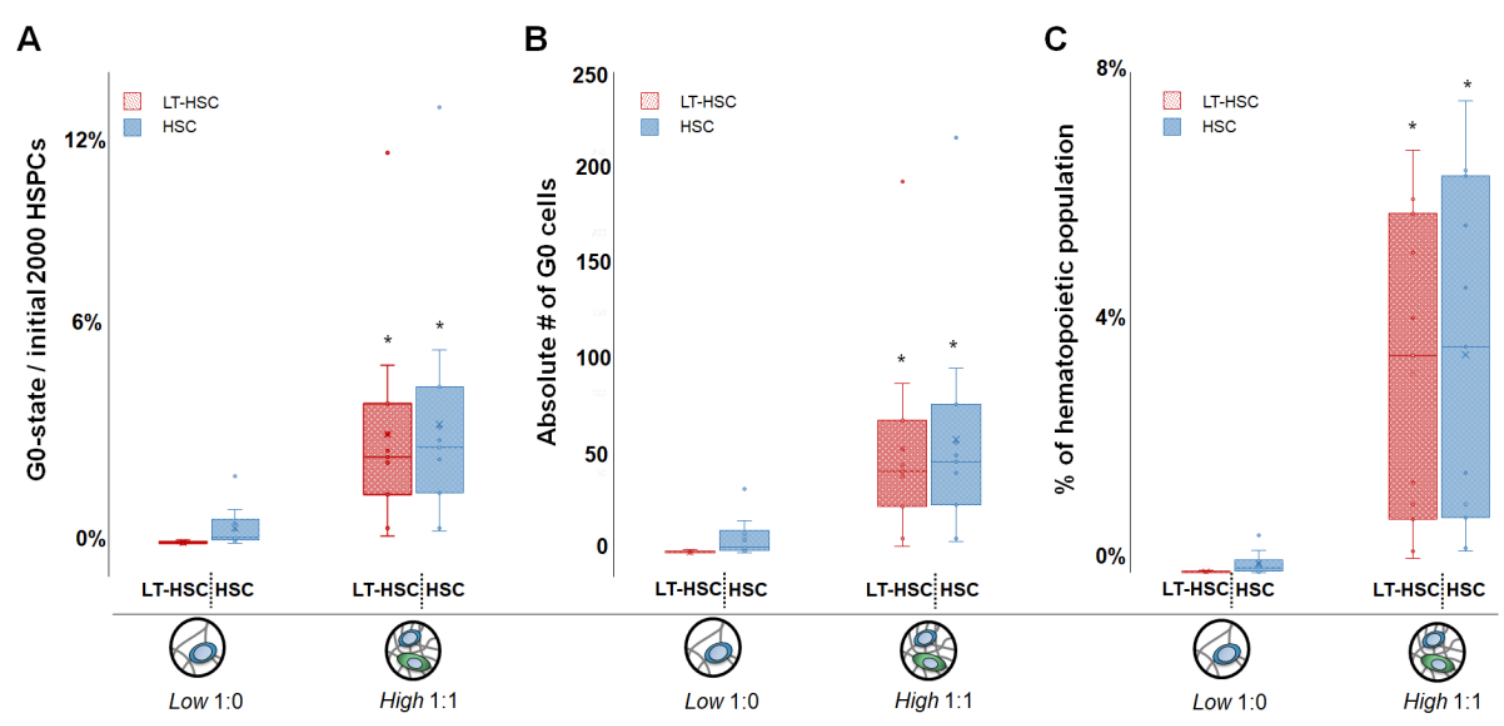

Figure 7. Comparison of LT-HSC and HSC quiescence after 7 days in conditions that led to the greatest maintenance of an HSC population when cultured in the absence (Low 1:0 HSPC:MSC) vs. presence (High 1:1 HSPC:MSC) of MSCs (gels=30, n=10). A) Fraction of quiescent LT-HSC or HSC, shown as a percentage of the initial 2000 HSPCs seeded per hydrogel condition. B) Absolute number of quiescent (G0) LT-HSC and HSCs within each sample. C) Percentage of quiescent cells calculated vs. the total number of hematopoietic lineage positive cells after 7 days in culture as a percentage of the overall hematopoietic lineage positive cell population. *: significantly different $(\mathrm{p}<0.05)$ versus the quiescence in high diffusivity hydrogel containing HSCs only (Low 1:0). 
Synthetic niche analogs for ex vivo culture of HSCs have begun to uncouple the complex signals that are inherent to the native HSC niche. Traditional efforts have demonstrated that changes in matrix composition, mechanics, and selective inclusion of growth factors can substantially alter HSC maintenance and differentiation patterns. Notably, selective interactions with fibronectin (46-48), or immobilization of SCF within a hydrogel (49-51), can enhance HSC maintenance. More recently, the concept of cell-cell signaling and its role on HSC lineage specification have begun to be examined, primarily in liquid (unhindered-diffusion) experimental systems. Csaszar et al. demonstrated that HSC-secreted cytokines can alter ex vivo expansion of HSCs within a bioreactor (52), while Müller et al. demonstrated that individual HSCs cultured in microcavities with autocrine feedback led to increased HSC quiescence (53). However, it is also important to consider the influence of heterotypic cell-cell interactions within more complex niche mimics. For this, 3D hydrogel biomaterials are advantageous as heterotypic cultures can be maintained in an environment where the kinetics of HSC-generated autocrine feedback and paracrine signaling from niche-associated cells can be manipulated by the poroelastic properties of the hydrogel itself. Our use of a GelMA hydrogel enables robust control over both mechanical cues and transport of soluble factors to impact HSC lineage and quiescence. Previously, we employed a collagen hydrogel system to examine feedback between HSCs and their progeny of lineage positive cells (31). That system demonstrated that signaling modes, i.e. paracrine vs autocrine, in a 3D environment impacted HSC fate decisions, with paracrine signals from Lin $^{+}$ hematopoietic cells capable of enhancing HSC myeloid, while the effects could be tempered in a diffusion-limited hydrogel environment promoted autocrine feedback and HSC maintenance (31). 
Importantly, while a hydrogel matrix facilitates studies of the influence of mechanical and heterotypic soluble signaling on HSC activity, it may also be sensitive to cell-mediated remodeling. The novelty of this work is the systematic approach to demonstrate the coordinated effects of matrix poroelastic properties, cell mediated remodeling, and heterotypic cell-cell interaction on HSC culture and quiescence. Concepts of dynamic reciprocity, often employed in the context of wound remodeling or the tumor microenvironment $(54,55)$, offer a novel avenue to explore the design of HSC culture platforms. Therefore, we developed a culture system that takes advantage of paracrine signaling and matrix remodeling from niche-associated mesenchymal stromal cells (MSCs). Our application of a library of GelMA hydrogels that span a range of mechanical properties inspired by the native HSC niche (16), as well as advanced poroelastic characterization methods $(56,57)$, allows us to examine dynamic relationships between MSCs, the local matrix architecture, and autocrine vs. paracrine domains. HSPCs were co-cultured with MSCs at varying ratios (1:0, 1:1, 1:10 HSPCs:MSCs; 2000 HSPCs) to demonstrate that HSC differentiation patterns can be manipulated by combinations of initial hydrogel properties, MSC mediated remodeling, and the balance of autocrine feedback versus MSC-generated paracrine signals.

Gelatin was chosen as the base material of our system given its inherent ability for cell interaction. It possesses RGD adhesion sites, along with cleavage sites for matrix metalloproteinases produced by MSCs (58). Methacrylamide-functionalization and subsequent crosslinking of GelMA reduces the risk of gelatin thermos-instability at elevated temperatures. The mechanical properties of the GelMA hydrogels encompassed the range of moduli observed in the native bone marrow niche (Fig. 2). In terms of biotransport, the Low and Med versus High 
hydrogels displayed two subsets of diffusion and mesh size. Previous results from Mahadik et al. (31), suggest that the poor diffusivity of the High hydrogel is characterized by a small radius of cell-secreted biomolecular diffusion, generating an autocrine-dominated regime for HSC culture (59). Similarly, the significantly increased diffusivity of the Low and Med hydrogels suggests a regime for HSC culture characterized by a large radius of cell-secreted diffusion that reduces the effects of autocrine feedback and enhances MSC-generated paracrine signaling. Dilute cell seeding densities were specifically chosen for this study to promote large cell to cell distances (86-205 $\mu \mathrm{m}$ of spacing between cells, assuming a simple cubic unit). The diffusivity of the gelatin constructs and the dilute seeding conditions (2000 HSPCs/hydrogel) restricted cell-cell interactions to soluble signaling, highlighting the role the hydrogel variants play in determining long-range cell communication.

We observed HSC population maintenance was strongly influenced by initial hydrogel poroelastic properties, the presence of MSCs, and MSC-associated remodeling processes. When cultured in purely homotypic environments, HSC maintenance was greatest in a high diffusion, low stiffness hydrogel (Low) (Fig. 5). To explore the influence of paracrine signals from nicheassociated MSCs, we subsequently examined HSC lineage specification patterns in heterotypic cultures in the same set of Low, Med, and High hydrogels with MSCs at either $1 \times 10^{5}$ or $1 \times 10^{6}$ MSCs/mL (1:1, 1:10 HSPCs:MSCs). The addition of cells did not significantly alter the initial Young's modulus (Fig. 3). The presence of MSCs yielded significant, dynamic matrix remodeling as seen via downregulated matrix deposition related genes $(\operatorname{Col} 1(\alpha 1)$, TIMP-1, TIMP-2, TIMP-3) and upregulated matrix degradation genes (MMP-2, MMP-9) (Fig. 4). Together, these results suggest dynamic changes in mesh size and biotransport. While we 
observed significant matrix remodeling via compressive testing in High hydrogels seeded with the highest MSC density, no clear trends were observed in the Low or Med conditions. Recent results from Schultz et al. described non-uniform matrix remodeling (60) resulting from differences in diffusive path lengths and reaction times for MMPs vs. TIMPs. This suggests remodeling of our hydrogels was likely non-uniform, making it difficult to resolve local remodeling processes within the heterotypic HSC-MSC cultures with bulk measurements of modulus. However, the significant changes in HSC activity observed as a result of co-culture of MSCs suggest dynamic interaction between HSCs and MSCs is an exciting avenue for further exploration.

The addition of large populations of MSCs (1:10 HSPCs:MSCs) led to a decrease in HSC maintenance for all hydrogel variants. This suggests oversaturation by MSCs likely leads to not only increased soluble signaling but also significantly greater remodeling, both of which reduce maintenance of early hematopoietic progenitors compared to HSC-only cultures (Fig. 5). However, addition of an equivalent amount of MSCs (1:1 HSPCs:MSCs) leads to a very different shift in HSC population dynamics, with an overwhelming increase in the ability to maintain an HSC population, most notably an increased fraction of quiescent HSCs which is suggested to be protective of long-term hematopoietic homeostasis in vivo, and valuable in culture systems for HSC expansion or lineage specification (61-63). This increase aligns with literature, confirming the ability of MSCs to influence HSC equilibrium through secreted factors within the in vivo niche(33). Particularly, the highest maintenance of HSCs in the 1:1 HSPCs:MSCs culture was observed in the High hydrogel variant that displayed minimal initial biotransport capabilities but also long-term signatures of MSC-mediated remodeling (MMP-9 
expression). This suggests the importance of a balance between initial autocrine-dominated feedback followed by MSC paracrine signals for increasing HSC maintenance. These findings highlight the possibility for selectively employing more complex culture systems to engineer HSC lineage specification patterns.

Bone marrow niches contain a complex milieu of external signals that play a significant role in mediating the activity of HSCs in vivo. The development of a synthetic niche analog for HSC culture can take advantage of poroelastic properties to mediate the balance of heterotypic cell signaling via soluble factors. Here, we explored the use of MSC-secreted paracrine signaling as well as MSC-mediated matrix remodeling as a means to increase HSC maintenance during in vitro culture using a library of GelMA hydrogels. Examining HSC differentiation and cell-cycle patterns, as well as dynamic remodeling of a model GelMA hydrogel in the presence of MSCs suggests a path towards ex vivo expansion of HSCs. We show that combinations of initial hydrogel properties and a moderate amount of MSCs increases the maintenance of HSCs in a culture system with initially hindered diffusion. These findings suggest that MSC-secreted paracrine signaling and initial autocrine feedback are essential in vitro tools seeking to expand HSC populations. Such findings offer exciting opportunities to explore concepts of dynamic reciprocity in the context of heterotypic cell cultures. Matrix remodeling as well as selective domains of autocrine feedback versus paracrine signaling can be leveraged for the intelligent design of an in vitro HSC culture systems.

\section{Materials and Methods}

\subsection{Material characterization}




\subsubsection{GelMA synthesis and hydrogel fabrication}

Porcine Type A gelatin (Sigma, St. Louis, MO) was functionalized along its backbone with pendulant methacrylamide groups following a previously described protocol $(58,64)$. In brief, methacrylate anhydride (MA) was added dropwise to a solution of gelatin in PBS before quenching in excess PBS, purification via dialysis, and lyophilization. The ratio of gelatin to MA was tuned to control the degree of functionalization $\left(35 \%, 50 \%, 85 \%\right.$ DOF; quantified by $\mathrm{H}^{1}$ NMR) (58). Methacrylamide-functionalized gelatin (GelMA) hydrogel variants were subsequently formed from $20 \mu \mathrm{L}$ of GelMA precursor suspension mixed with a lithium acylphosphinate (LAP) photoinitiator (PI) in circular Teflon molds (5mm dia.) exposed to 7.14 $\mathrm{mW} / \mathrm{cm}^{2} \mathrm{UV}$ light for 30 seconds. After crosslinking, hydrogels were immediately hydrated in $\operatorname{PBS}(49,65)$.

\subsubsection{Determination of poroelastic properties}

A library of 18 acellular hydrogels were fabricated from 35, 50, and 85\% DOF GelMA at 4, 5, and 7.5\% (w/v) GelMA, with 0.05 or $0.1 \%(w / v) ~ P I$. The Young's modulus was determined using an Instron 5943 mechanical tester in unconfined compression (Instron, Norwood, MA). Briefly, a $0.005 \mathrm{~N}$ preload was applied followed by compression at a rate of $0.1 \mathrm{~mm} / \mathrm{min}$ until $20 \%$ compression was reached. The Young's modulus was taken as the slope of the linear fit applied to $10 \%$ of the stress vs. strain data using Origin Statistical Software (Northampton, MA) $(66,67)$. An offset of 0 or $5 \%$ strain was applied prior to linear fit analysis to confirm that the compression platen was completely in contact with the hydrogel surface. The elastic moduli of cellular hydrogels containing $1 \times 10^{5}$ or $1 \times 10^{6} \mathrm{MSC} / \mathrm{mL}$ (experimental description below) were traced over seven days in culture, using the same compression protocol. After mechanical 
analysis, MSC-hydrogels were immediately put on dry ice and stored at $-80^{\circ} \mathrm{C}$ for subsequent gene expression analyses.

Stress-relaxation indentation tests were performed on acellular hydrogels using MFP-3D AFM (Asylum Research, Goleta, CA), with a $4.5 \mu \mathrm{m}$ spherical tip (Novascan, Ames, IA). The generated force curve was used to extract out the shear modulus and diffusion coefficient of water. Specimens were rapidly indented to $0.5,1$, or $2 \mu \mathrm{m}$, and held for a test time of 5 seconds. This process has been described elsewhere, but in brief, the instantaneous force was normalized to the far-field force and fitted to a finite element analysis formula by modulating the characteristic time and material properties (shear modulus, diffusion) $(56,57,68,69)$.

\subsubsection{Mesh size estimate}

The mesh size of the hydrogel library was determined using hydrogel swelling ratios and is a function of the volume fraction and the mean-squared end-to-end distance of the gelatin chain (70-72).

(1) $\quad \xi=v_{2 s}^{-\frac{1}{3}} *\left(l\left(2 \frac{M_{c}}{M_{r}}\right) C_{n}\right)^{\frac{1}{2}}$

In determining material parameters, it was assumed that the gelatin chains were long enough to neglect chain-end effects. It was also assumed that the molecular weight of the repeat unit, $M_{r}$, was the averaged molecular weight of the amino acid composition. Amino Acid Analysis was performed at the UC Davis Genome Center with a Hitachi L-8900 (Li-based) analyzer. The mass swelling ratio was determined by the ratio of the dry to wet weight. The swelling ratio was determined immediately following crosslinking or after $24 \mathrm{hrs}$ in PBS, respectively known as relaxed and equilibrium states. Hydrogels were weighed before and after 
lyophilization to obtain the mass swelling ratio, and then transformed to volumetric swelling ratio following the equation below, using the solvent density $\left(\rho_{P B S}=1.01 \mathrm{~g} / \mathrm{cm}^{3}\right)(73)$ and gelatin density $\left(\rho_{\text {gelatin }}=1.345 \mathrm{~g} / \mathrm{cm}^{3}\right)(74-76)$.

$$
Q_{v}=1+\frac{\rho_{p}}{\rho_{s}}\left(Q_{m}-1\right)
$$

From this, the volume fraction of gelatin is calculated as:

$$
v=\frac{1}{Q_{v}}
$$

The molecular weight between crosslinks, $M_{c}$, was estimated using the well-known Flory-Rehner equation adapted by Bray and Merrill for use in polymers crosslinked in solvent $(71,77)$. The applicability of the modified equation for specific concentration regimes was confirmed by Peppas and Merrill (70). The $M_{c}$ is a function of material properties such as the molar weight of the solvent, $V_{1}$, the specific volume of the polymer, $\bar{v}$, and the solvent-polymer interaction, $X_{1}$, also known as Flory's Chi parameter. It is also a function of the system parameters such as the volume fraction of the polymer in its relaxed state and in the equilibrium state, $v_{2 r}$ and $v_{2 s}$, and the number average molecular weight before crosslinking, $M_{n}$.

$$
\frac{1}{M_{c}}=\frac{2}{M_{n}}-\frac{\frac{\bar{v}}{V_{1}}\left[\ln \left(1-v_{2 s}\right)+v_{2 s}+X_{1} v_{2 s}^{2}\right]}{v_{2 r}\left[\left(\frac{v_{2 s}}{v_{2 r}}\right)^{\frac{1}{3}}-\frac{1}{2}\left(\frac{v_{2 s}}{v_{2 r}}\right)\right]}
$$

Flory's characteristic ratio, $C_{n}$, can be approximated by a constant number as the number of monomers goes to infinity, $\lim _{n \rightarrow \infty} C_{n}=C_{\infty}$. For a stiff chain, this can be approximated using the worm-like or Kratky-Porod persistence model by using the persistence length, $l_{p}$, and the monomer unit length, and modified for proteins $(78,79)$ :

$$
C_{\infty}=0.95 \frac{2 l_{p}}{l}
$$

\subsection{Hematopoietic stem cell isolation}


All work involving primary cell extraction was conducted under approved animal welfare protocols (Institutional Animal Care and Use Committee, University of Illinois at UrbanaChampaign). Primary murine hematopoietic stem progenitor cells (HSPC) were extracted from the femur and tibia bone marrow of C57BL/6 female mice, age $4-8$ weeks (Jackson Labs). The bones were gently crushed with pestle and mortar and washed with PBS $+5 \%$ FBS prior to filtration with a $40 \mu \mathrm{m}$ sterile filter $(31,49)$. The collected suspension was lysed with ACK lysis buffer (Invitrogen, Carlsbad, CA), and then rinsed with PBS/FBS. Initial HSPC isolation was performed using defined protocols of EasySep ${ }^{\mathrm{TM}}$ Mouse Hematopoietic Progenitor Cell Enrichment Kit and Magnet (\#19756,\#18000, Stemcell Technologies, CA). A secondary isolation further enriched the HSPC population by collecting the $\mathrm{Lin}^{-} \mathrm{Sca}^{-}{ }^{+} \mathrm{c}-\mathrm{kit}^{+}$(LSK) fraction using a BD FACS Aria II flow cytometer. All antibodies were supplied by eBioscience (San Diego, CA), and are as follows: PE-conjugated Sca-1 (1:100 dilution), APC-efluor780conjugated c-kit (1:100 dilution), and a cocktail of FITC-conjugated lineage specific surface markers (CD5, B220, Mac-1, CD8a, Gr-1, and Ter-119) (80-82). The LSK faction was collect in PBS $+25 \%$ FBS on ice and immediately used $(13,21,31,49,83-85)$.

\section{Mesenchymal stromal cell isolation}

Murine MSCs were isolated from the femur and tibia bones used for HSPC extraction. Following gentle crushing with a pestle and mortar, MSCs were isolated from bone following commercially available defined protocols of MesenCult ${ }^{\mathrm{TM}}$ Proliferation Kit with MesenPure ${ }^{\mathrm{TM}}$ (\#05512 Stemcell Technologies, CA). The MSCs were cultured in one T25 flask per mouse and allowed to expand for two weeks prior to collection (zero passage), with a half-media change after one week. Non-adherent cells were washed away, and the adherent cells were collected 
after trypsinization with Gibco TrypLE (ThermoFisher Scientific, Waltham, MA). Cells were collected and immediately used.

\subsection{Hematopoietic stem cell culture in GelMA hydrogel in the presence of MSCs}

Single culture HSPCs were encapsulated in GelMA hydrogels at a density of $1 \times 10^{5}$ HSPCs/mL. Similarly, co-cultures of HSPCs and MSCs were encapsulated at a ratio of 1:1 or 1:10, HSPCs to MSC, keeping a constant density of $1 \times 10^{5} \mathrm{HSPCs} / \mathrm{mL}$. HSPCs or MSCs in a suspension were added to the hydrogel precursor solution at the desired seeding density of $1 \times 10^{5}$ HSPCs $/ \mathrm{mL}$ and $1 \times 10^{5}$ or $1 \times 10^{6} \mathrm{MSCs} / \mathrm{mL}$, while maintaining the concentration of GelMA. Upon crosslinking, each hydrogel-cell construct was placed into an individual well of a 48-well plate, and cultured at $37^{\circ} \mathrm{C}\left(5 \% \mathrm{CO}_{2}\right)$ for 7 days in $300 \mu \mathrm{L}$ StemSpan ${ }^{\mathrm{TM}}$ SFEM (\#09650 Stemcell Technologies) supplemented with 100 ng/mL SCF (Peprotech) and 0.1\% Pen Strep (Gibco), with media changes every 2 days (21).

\subsection{Analysis of HSC differentiation patterns}

Differentiation patterns of HSPCs were analyzed via Fluorescence-Assisted Cytometry (FACs), using a BD LSR Fortessa (BD Biosciences, San Jose, CA). Cell seeded hydrogels were degraded with 100 Units of Collagenase Type IV (Worthington Biochemical, Lakewood, NJ) for 20 minutes at $37^{\circ} \mathrm{C}$, and gently mixed via pipetting every 10 minutes. The reaction was

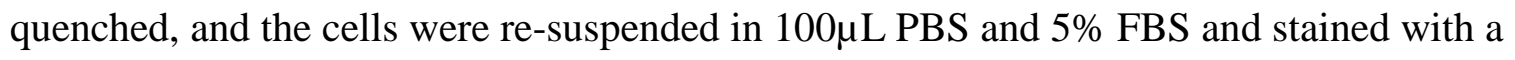
cocktail of antibodies (eBioscience, San Diego, CA) to profile HSC lineage specification: eFluor 660 CD34 (1:100 dilution, 90 mins), PerCP-eFluor 710 CD135 (1:100 dilution, 30 mins), eFluor 450 CD16/CD32 (1:200 dilution, 30 mins), APC-eFluor 780 c-Kit (1:800 dilution, 30 mins), PE 
Sca-1 (1:400 dilution, 30 mins), FITC lineage cocktail (CD5, B220, Mac-1, CD8z, Gr-1, Ter-

119, 1:100 dilution, $30 \mathrm{~min}$ ). Hematopoietic cells were subsequently classified as more primitive Long-Term repopulating HSCs (LT-HSCs: CD34 $\left.{ }^{\text {lo }} \mathrm{CD} 135^{\mathrm{lo}} \mathrm{Lin}^{-} \mathrm{Sca}^{+} \mathrm{c}^{\mathrm{k}} \mathrm{kit}{ }^{+}\right)(84,86,87)$; differentiated Short-Term repopulating HSCs (ST-HSCs: CD34 ${ }^{\text {hi }}$ CD135 $5^{\text {lo }}$ LSK) $(84,86,87)$; more differentiated Multipotent progenitors (MPPs: $\left.\mathrm{CD}^{+} 4^{+} \mathrm{CD} 135^{+} \mathrm{LSK}\right)(87,88)$, and lineagespecified Common Myeloid Progenitors (CMPs: Lin ${ }^{-}$c-kit ${ }^{+} \mathrm{Sca}^{-}$CD34+ CD16/32-) (88), (81, 89-91). Individual cell populations are reported as percentages of the total hematopoietic cell population in each culture, with terminal cells classified as hematopoietic cells that were not part of the LSK fraction. For some cell population analyses, LT-HSC and ST-HSC were grouped together as a common HSC population.

\subsection{Analysis of cell cycle state}

Conditions identified as optimal in maintaining HSC numbers (HSC only in Low hydrogel variant; 1:1 HSC:MSC co-culture in High hydrogel variant) were chosen for subsequent cell cycle analysis. The 1:0 Low and 1:1 High (HSPC:MSC) conditions were cultured and cells were isolated at day 7 as described above. Experimental replicates ( $n=10 /$ condition) were performed; each sample contained three separately cultured hydrogels $\left(\mathrm{n}_{\mathrm{gels}}=30\right)$. Isolated cells were then live/dead stained with LIVE/DEAD ${ }^{\text {TM }}$ Fixable Red Dead Cell Stain Kit (\#L34971, ThermoFisher) following the standard protocol, and LT-HSC, ST-HSC, and MPP populations labeled via antibody cocktail (defined above). Stained cells were fixed and permeabilized with Foxp3 / Transcription Factor Staining Buffer Set (\#00-5523-00, eBioscience), and stained with BV605-conjugated Ki-67 (1:100 dilution) and DAPI (\#D21490, ThermoFisher). Quiescent cells (DAPI ${ }^{2 n} \mathrm{Ki}^{-67^{-}}$) from each hematopoietic population were 
subsequently analyzed via Flow Cytometry.

\subsection{Analysis of MSC mediated-remodeling profiles}

Matrix remodeling associated gene expression profiles were examined for MSC-seeded hydrogel specimens via QuantiStudio 7 Flex real-time PCR (Applied Biosystems). Frozen hydrogels isolated at days $0,1,4$, and 7 during culture were crushed using a pestle, with mRNA extracted using RNeasy Plant Mini Kit (Qiagen, Germany). mRNA to cDNA was accomplished using the QuantiTect Rev. Transcription kit (Qiagen) and prepped for RT-PCR using PIPETMAX (Gilson, Middleton, WI). The $\mathrm{C}_{\mathrm{T}}$ of each gene/sample was performed in duplicate. Results were analyzed using the comparative CT method $\left(2^{-\Delta \Delta C T}\right)$. Primer specificity and efficiency were validated prior to comparative $\mathrm{C}_{\mathrm{T}}$ analysis (92). Matrix deposition genes examined were collagen, type I, alpha 1 (Col1a1), and tissue inhibitors of metalloprotease TIMP1, TIMP-2, and TIMP-3. Matrix degradation genes examined were matrix metalloproteases MMP-2 and MMP-9, also known as Gelatinase-A and Gelatinase-B (93). Expression of glyceraldehyde-3-phosphate dehydrogenase (GAPDH) was used for housekeeping. Gene expression was first normalized to the housekeeping gene (GAPDH), and then compared against the gene expression at day 0 of each specific seeding and hydrogel condition (Fig. S4). Primer information can be found in Table S1.

\subsection{Statistics}

All statistics were performed on a minimum of 3 replicates. Significance was tested with Origin Statistical Software using 2-way ANOVA, at a significance level of 0.05. Normality of the data was determined using the Shapiro-Wilkes test at significance 0.05 (94), and 
homoscedasticity (equality of variance) determined using Levene's Test (95). Significance of quiescence was determined with the nonparametric Mann-Whitney test (2-sample) at the 0.05 significance level. Post-hoc power analysis was used to confirm the sample size was large enough to maintain a maximum Type I error of $\alpha=0.05$, and Type II error of $\beta=0.20(96,97)$. Young's modulus outliers were removed using Dixon's Q-test at significance level 0.05 (98). Means are reported with their associated standard deviations. In the comparative $\mathrm{C}_{\mathrm{T}}$ method of gene expression, the error was propagated throughout the calculations.

\section{Supplementary Materials}

Fig. S1. Compressive mechanical analysis of the complete library of hydrogels.

Fig. S2. Shear modulus characterization of the complete library of hydrogels.

Fig. S3. The diffusion coefficient of water in each hydrogel was measured for the complete library of hydrogels.

Fig. S4. Relative expression of matrix-associated genes in Low, Med, and High hydrogels seeded with $1 \times 10^{5}(1: 1)$ and $1 \times 10^{6} \mathrm{MSCs} / \mathrm{mL}(1: 10)$

Fig. S5. Relative expression of matrix-associated genes in Low, Med, and High hydrogels seeded with $1 \times 10^{5}(1: 1)$ and $1 \times 10^{6} \mathrm{MSCs} / \mathrm{mL}(1: 10)$

Fig. S6. Hematopoietic cell differentiation patterns within Low, Med, and High hydrogels with 0, $1 \times 10^{5}$, and $1 \times 10^{6} \mathrm{MSCs} / \mathrm{mL}(1: 0,1: 1$, and 1:10 respectively).

Fig. S7. Number and fraction of quiescent LT-HSCs and HSCs in liquid culture containing either $1 \times 10^{5} \mathrm{HSPCs} / \mathrm{mL}$ alone $(1: 0)$, or a mixture of $1 \times 10^{5} \mathrm{HSPCs} / \mathrm{mL}$ and $1 \times 10^{5} \mathrm{MSCs} / \mathrm{mL}(1: 1)$

Table S1. Forward and reverse primer sequences for matrix associated genes.

Table S2. Parameters for hydrogel mesh calculations. 
bioRxiv preprint doi: https://doi.org/10.1101/289553; this version posted February 13, 2019. The copyright holder for this preprint (which was not certified by peer review) is the author/funder. All rights reserved. No reuse allowed without permission. 


\section{References and Notes:}

1. S. Doulatov, F. Notta, E. Laurenti, J. E. Dick, Hematopoiesis: a human perspective. Cell Stem Cell 10, 120136 (2012).

2. A. Mendelson, P. S. Frenette, Hematopoietic stem cell niche maintenance during homeostasis and regeneration. Nat Med 20, 833-846 (2014).

3. M. Ogawa, Differentiation and proliferation of hematopoietic stem cells. Blood 81, 2844-2853 (1993).

4. E. M. Pietras, M. R. Warr, E. Passegue, Cell cycle regulation in hematopoietic stem cells. J Cell Biol 195, 709-720 (2011).

5. A. Wilson, A. Trumpp, Bone-marrow haematopoietic-stem-cell niches. Nat Rev Immunol 6, 93-106 (2006).

6. S. J. Morrison, D. T. Scadden, The bone marrow niche for haematopoietic stem cells. Nature 505, $327-334$ (2014).

7. G. B. Adams, D. T. Scadden, The hematopoietic stem cell in its place. Nat Immunol 7, $333-337$ (2006).

8. $\quad$ L. E. Purton, D. T. Scadden, in StemBook. (Cambridge (MA), 2008).

9. D. S. Krause, D. T. Scadden, F. I. Preffer, The hematopoietic stem cell niche--home for friend and foe? Cytometry B Clin Cytom 84, 7-20 (2013).

10. E. A. Copelan, Hematopoietic stem-cell transplantation. N Engl J Med 354, 1813-1826 (2006).

11. A. D'Souza, X. Zhu. (2016).

12. J. Zhang, L. Li, Stem cell niche: microenvironment and beyond. J Biol Chem 283, 9499-9503 (2008).

13. J. S. Choi, B. P. Mahadik, B. A. Harley, Engineering the hematopoietic stem cell niche: Frontiers in biomaterial science. Biotechnol J 10, 1529-1545 (2015).

14. C. Nombela-Arrieta et al., Quantitative imaging of haematopoietic stem and progenitor cell localization and hypoxic status in the bone marrow microenvironment. Nat Cell Biol 15, 533-543 (2013).

15. S. K. Nilsson et al., Immunofluorescence characterization of key extracellular matrix proteins in murine bone marrow in situ. J Histochem Cytochem 46, 371-377 (1998).

16. L. E. Jansen, N. P. Birch, J. D. Schiffman, A. J. Crosby, S. R. Peyton, Mechanics of intact bone marrow. J Mech Behav Biomed Mater 50, 299-307 (2015). 
17. J. Holst et al., Substrate elasticity provides mechanical signals for the expansion of hemopoietic stem and progenitor cells. Nat Biotechnol 28, 1123-1128 (2010).

18. C. Lee-Thedieck, N. Rauch, R. Fiammengo, G. Klein, J. P. Spatz, Impact of substrate elasticity on human hematopoietic stem and progenitor cell adhesion and motility. J Cell Sci 125, 3765-3775 (2012).

19. M. Krater et al., Bone marrow niche-mimetics modulate HSPC function via integrin signaling. Sci Rep 7, 2549 (2017).

20. J. A. LaIuppa, T. A. McAdams, E. T. Papoutsakis, W. M. Miller, Culture materials affectex vivo expansion of hematopoietic progenitor cells. Journal of Biomedical Materials Research 36, 347-359 (1997).

21. J. S. Choi, B. A. Harley, Marrow-inspired matrix cues rapidly affect early fate decisions of hematopoietic stem and progenitor cells. Sci Adv 3, e1600455 (2017).

22. A. Janowska-Wieczorek, M. Majka, J. Ratajczak, M. Z. Ratajczak, Autocrine/paracrine mechanisms in human hematopoiesis. Stem Cells 19, 99-107 (2001).

23. M. Majka et al., Numerous growth factors, cytokines, and chemokines are secreted by human CD34(+) cells, myeloblasts, erythroblasts, and megakaryoblasts and regulate normal hematopoiesis in an autocrine/paracrine manner. Blood 97, 3075-3085 (2001).

24. T. Stylianopoulos et al., Diffusion of particles in the extracellular matrix: the effect of repulsive electrostatic interactions. Biophys J 99, 1342-1349 (2010).

25. P. Kamali-Zare, C. Nicholson, Brain extracellular space: geometry, matrix and physiological importance. Basic Clin Neurosci 4, 282-286 (2013).

26. A. Pluen, P. A. Netti, R. K. Jain, D. A. Berk, Diffusion of Macromolecules in Agarose Gels: Comparison of Linear and Globular Configurations. Biophysical Journal 77, 542-552 (1999).

27. C. C. van Donkelaar, G. Chao, D. L. Bader, C. W. Oomens, A reaction-diffusion model to predict the influence of neo-matrix on the subsequent development of tissue-engineered cartilage. Comput Methods Biomech Biomed Engin 14, 425-432 (2011).

28. E. Muller et al., Distinguishing autocrine and paracrine signals in hematopoietic stem cell culture using a biofunctional microcavity platform. Sci Rep 6, 31951 (2016).

29. S. Taqvi, L. Dixit, K. Roy, Biomaterial-based notch signaling for the differentiation of hematopoietic stem cells into T cells. J Biomed Mater Res A 79, 689-697 (2006). 
30. E. Csaszar et al., Rapid expansion of human hematopoietic stem cells by automated control of inhibitory feedback signaling. Cell Stem Cell 10, 218-229 (2012).

31. B. P. Mahadik, N. A. Bharadwaj, R. H. Ewoldt, B. A. Harley, Regulating dynamic signaling between hematopoietic stem cells and niche cells via a hydrogel matrix. Biomaterials 125, 54-64 (2017).

32. A. Ehninger, A. Trumpp, The bone marrow stem cell niche grows up: mesenchymal stem cells and macrophages move in. J Exp Med 208, 421-428 (2011).

33. S. Mendez-Ferrer et al., Mesenchymal and haematopoietic stem cells form a unique bone marrow niche. Nature 466, 829-834 (2010).

34. B. A. Anthony, D. C. Link, Regulation of hematopoietic stem cells by bone marrow stromal cells. Trends Immunol 35, 32-37 (2014).

35. T. Li, Y. Wu, Paracrine molecules of mesenchymal stem cells for hematopoietic stem cell niche. Bone Marrow Res 2011, 353878 (2011).

36. W. Wagner et al., Molecular and secretory profiles of human mesenchymal stromal cells and their abilities to maintain primitive hematopoietic progenitors. Stem Cells 25, 2638-2647 (2007).

37. S. El Marsafy, Mesenchymal Stem Cells: Pivotal Players in Hematopoietic Stem Cell Microenvironment. Journal of Stem Cell Research \& Therapy 04, (2014).

38. T. Sugiyama, H. Kohara, M. Noda, T. Nagasawa, Maintenance of the hematopoietic stem cell pool by CXCL12-CXCR4 chemokine signaling in bone marrow stromal cell niches. Immunity 25, 977-988 (2006).

39. C. Mirantes, E. Passegue, E. M. Pietras, Pro-inflammatory cytokines: emerging players regulating HSC function in normal and diseased hematopoiesis. Exp Cell Res 329, 248-254 (2014).

40. A. Tocci, L. Forte, Mesenchymal stem cell: use and perspectives. Hematol J 4, 92-96 (2003).

41. G. S. Schultz, J. M. Davidson, R. S. Kirsner, P. Bornstein, I. M. Herman, Dynamic reciprocity in the wound microenvironment. Wound Repair Regen 19, 134-148 (2011).

42. J. Alexander, E. Cukierman, Stromal dynamic reciprocity in cancer: intricacies of fibroblastic-ECM interactions. Curr Opin Cell Biol 42, 80-93 (2016).

43. A. Page-McCaw, A. J. Ewald, Z. Werb, Matrix metalloproteinases and the regulation of tissue remodelling. Nat Rev Mol Cell Biol 8, 221-233 (2007). 
44. C. Sassoli et al., Defining the role of mesenchymal stromal cells on the regulation of matrix metalloproteinases in skeletal muscle cells. Exp Cell Res 323, 297-313 (2014).

45. W. P. Daley, S. B. Peters, M. Larsen, Extracellular matrix dynamics in development and regenerative medicine. J Cell Sci 121, 255-264 (2008).

46. J. S. Choi, B. A. C. Harley, Marrow-inspired matrix cues rapidly affect early fate decisions of hematopoietic stem and progenitor cells. Science Advances 3, e1600455 (2017).

47. J. S. Choi, B. A. C. Harley, Substrate elasticity and chmistry regulate the biophysical properties and lineage commitment of hematopoietic stem cells. In preparation, (2013).

48. E. Altrock, C. A. Muth, G. Klein, J. P. Spatz, C. Lee-Thedieck, The significance of integrin ligand nanopatterning on lipid raft clustering in hematopoietic stem cells. Biomaterials 33, 3107-3118 (2012).

49. B. P. Mahadik, S. Pedron Haba, L. J. Skertich, B. A. Harley, The use of covalently immobilized stem cell factor to selectively affect hematopoietic stem cell activity within a gelatin hydrogel. Biomaterials 67, 297 307 (2015).

50. M. L. Cuchiara et al., Bioactive poly(ethylene glycol) hydrogels to recapitulate the HSC niche and facilitate HSC expansion in culture. Biotechnol Bioeng 113, 870-881 (2016).

51. M. L. Cuchiara, K. L. Horter, O. A. Banda, J. L. West, Covalent immobilization of stem cell factor and stromal derived factor 1alpha for in vitro culture of hematopoietic progenitor cells. Acta Biomater 9, 92589269 (2013).

52. E. Csaszar et al., Rapid expansion of human hematopoietic stem cells by automated control of inhibitory feedback signaling. Cell Stem Cell 10, 218-229 (2012).

53. E. Müller et al., Distinguishing autocrine and paracrine signals in hematopoietic stem cell culture using a biofunctional microcavity platform. Sci Rep 6, 31951 (2016).

54. J. Alexander, E. Cukierman, Stromal dynamic reciprocity in cancer: intricacies of fibroblastic-ECM interactions. Current Opinion in Cell Biology 42, 80-93 (2016).

55. G. S. Schultz, J. M. Davidson, R. S. Kirsner, P. Bornstein, I. M. Herman, Dynamic reciprocity in the wound microenvironment. Wound Repair and Regeneration 19, 134-148 (2011).

56. Z. I. Kalcioglu, R. Mahmoodian, Y. Hu, Z. Suo, K. J. Van Vliet, From macro- to microscale poroelastic characterization of polymeric hydrogels via indentation. Soft Matter 8, (2012). 
57. Y. Hu, X. Zhao, J. J. Vlassak, Z. Suo, Using indentation to characterize the poroelasticity of gels. Applied Physics Letters 96, (2010).

58. S. Pedron, B. A. Harley, Impact of the biophysical features of a 3D gelatin microenvironment on glioblastoma malignancy. J Biomed Mater Res A 101, 3404-3415 (2013).

59. S. Pedron et al., Patterning Three-Dimensional Hydrogel Microenvironments Using Hyperbranched Polyglycerols for Independent Control of Mesh Size and Stiffness. Biomacromolecules 18, 1393-1400 (2017).

60. K. M. Schultz, K. A. Kyburz, K. S. Anseth, Measuring dynamic cell-material interactions and remodeling during 3D human mesenchymal stem cell migration in hydrogels. Proc Natl Acad Sci U S A 112, E37573764 (2015).

61. J. Li, Quiescence regulators for hematopoietic stem cell. Exp Hematol 39, 511-520 (2011).

62. R. Aggarwal, J. Lu, V. J. Pompili, H. Das, Hematopoietic Stem Cells: Transcriptional Regulation, Ex Vivo Expansion and Clinical Application. Current Molecular Medicine 12, 34-49 (2012).

63. A. Nakamura-Ishizu, H. Takizawa, T. Suda, The analysis, roles and regulation of quiescence in hematopoietic stem cells. Development 141, 4656-4666 (2014).

64. J. A. Benton, C. A. DeForest, V. Vivekanandan, K. S. Anseth, Photocrosslinking of gelatin macromers to synthesize porous hydrogels that promote valvular interstitial cell function. Tissue Eng Part A 15, 32213230 (2009).

65. J. E. Chen, S. Pedron, B. A. C. Harley, The Combined Influence of Hydrogel Stiffness and Matrix-Bound Hyaluronic Acid Content on Glioblastoma Invasion. Macromol Biosci 17, (2017).

66. A. International. (ASTM International, 2017).

67. S. Pedron, E. Becka, B. A. Harley, Regulation of glioma cell phenotype in 3D matrices by hyaluronic acid. Biomaterials 34, 7408-7417 (2013).

68. Y. H. Hu, X. Chen, G. M. Whitesides, J. J. Vlassak, Z. G. Suo, Indentation of polydimethylsiloxane submerged in organic solvents. Journal of Materials Research 26, 785-795 (2011).

69. Y. Lai, Y. Hu, Unified solution for poroelastic oscillation indentation on gels for spherical, conical and cylindrical indenters. Soft Matter 13, 852-861 (2017). 
70. N. A. Peppas, E. W. Merrill, Crosslinked poly(vinyl alcohol) hydrogels as swollen elastic networks. Journal of Applied Polymer Science 21, 1763-1770 (1977).

71. J. C. Bray, E. W. Merrill, Poly(vinyl alcohol) hydrogels. Formation by electron beam irradiation of aqueous solutions and subsequent crystallization. Journal of Applied Polymer Science 17, 3779-3794 (1973).

72. T. Canal, N. A. Peppas, Correlation between mesh size and equilibrium degree of swelling of polymeric networks. J Biomed Mater Res 23, 1183-1193 (1989).

73. J. E. Schiel, D. S. Hage, Density measurements of potassium phosphate buffer from 4 to 45 degrees C. Talanta 65, 495-500 (2005).

74. I. G. Fels, Hydration and density of collagen and gelatin. Journal of Applied Polymer Science 8, 1813-1824 (1964).

75. J. H. Fessler, A. J. Hodge, Ultracentrifugal observation of phase transitions in density gradients. Journal of Molecular Biology 5, 446-IN442 (1962).

76. F. Anjum et al., Enzyme responsive GAG-based natural-synthetic hybrid hydrogel for tunable growth factor delivery and stem cell differentiation. Biomaterials 87, 104-117 (2016).

77. P. J. Flory, J. Rehner, Statistical Mechanics of Cross-Linked Polymer Networks II. Swelling. The Journal of Chemical Physics 11, 521-526 (1943).

78. W. Burchard, in Physical Techniques for the Study of Food Biopolymers. (1994), chap. Chapter 4, pp. 151213.

79. J. D. Bryngelson, E. M. Billings, in Physics of Biological Systems. (Springer, 1997), pp. 80-116.

80. G. A. Challen, N. Boles, K. K. Y. Lin, M. A. Goodell, Mouse Hematopoietic Stem Cell Identification and Analysis. Cytom Part A 75a, 14-24 (2009).

81. L. Yang et al., Identification of Lin(-)Sca1(+)kit(+)CD34(+)Flt3- short-term hematopoietic stem cells capable of rapidly reconstituting and rescuing myeloablated transplant recipients. Blood 105, 2717-2723 (2005).

82. S. Okada et al., Invivo and Invitro Stem-Cell Function of C-Kit-Positive and Sca-1-Positive Murine Hematopoietic-Cells. Blood 80, 3044-3050 (1992). 
83. B. P. Mahadik, T. D. Wheeler, L. J. Skertich, P. J. A. Kenis, B. A. C. Harley, Microfluidic Generation of Gradient Hydrogels to Modulate Hematopoietic Stem Cell Culture Environment. Advanced Healthcare Materials 3, 449-458 (2014).

84. A. E. Beaudin, S. W. Boyer, E. C. Forsberg, Flk2/Flt3 promotes both myeloid and lymphoid development by expanding non-self-renewing multipotent hematopoietic progenitor cells. Exp Hematol 42, 218-229 e214 (2014)

85. Y. Ilin, J. S. Choi, B. A. Harley, M. L. Kraft, Identifying States along the Hematopoietic Stem Cell Differentiation Hierarchy with Single Cell Specificity via Raman Spectroscopy. Anal Chem 87, 11317$11324(2015)$

86. L. P. Yang et al., Identification of Lin(-)Sca1(+)kit(+)CD34(+)Flt(3-) short-term hematopoietic stem cells capable of rapidly reconstituting and rescuing myeloablated transplant recipients. Blood 105, 2717-2723 (2005).

87. C. Tian, Y. Zhang, Purification of hematopoietic stem cells from bone marrow. Ann Hematol 95, 543-547 (2016).

88. Y. Zhang et al., Stress hematopoiesis reveals abnormal control of self-renewal, lineage bias, and myeloid differentiation in Mll partial tandem duplication (Mll-PTD) hematopoietic stem/progenitor cells. Blood 120, $1118-1129$ (2012).

89. G. A. Challen, N. Boles, K. K. Lin, M. A. Goodell, Mouse hematopoietic stem cell identification and analysis. Cytometry A 75, 14-24 (2009).

90. A. Mayle, M. Luo, M. Jeong, M. A. Goodell, Flow cytometry analysis of murine hematopoietic stem cells. Cytometry A 83, 27-37 (2013).

91. G. J. Spangrude, S. Heimfeld, I. L. Weissman, Purification and characterization of mouse hematopoietic stem cells. Science 241, 58-62 (1988).

92. in Part Number 4371095, Rev. B. (Applied Biosystems, 2008).

93. L. C. Abraham, J. F. Dice, K. Lee, D. L. Kaplan, Phagocytosis and remodeling of collagen matrices. Exp Cell Res 313, 1045-1055 (2007).

94. A. Ghasemi, S. Zahediasl, Normality tests for statistical analysis: a guide for non-statisticians. Int J Endocrinol Metab 10, 486-489 (2012). 
95. R. J. Carroll, H. Schneider, A note on levene's tests for equality of variances. Statistics \& Probability Letters 3, 191-194 (1985).

96. J. Cohen, Statistical power analysis for the behavioral sciences. (L. Erlbaum Associates, Hillsdale, N.J. :, ed. 2nd ed., 1988).

97. J. Cohen, A power primer. Psychol Bull 112, 155-159 (1992).

98. R. B. Dean, W. J. Dixon, Simplified Statistics for Small Numbers of Observations. Analytical Chemistry 23, 636-638 (1951).

99. J. Ye et al., Primer-BLAST: a tool to design target-specific primers for polymerase chain reaction. $B M C$ Bioinformatics 13, 134 (2012).

100. A. Spandidos et al., A comprehensive collection of experimentally validated primers for Polymerase Chain Reaction quantitation of murine transcript abundance. BMC Genomics 9, 633 (2008).

101. A. Spandidos, X. Wang, H. Wang, B. Seed, PrimerBank: a resource of human and mouse PCR primer pairs for gene expression detection and quantification. Nucleic Acids Res 38, D792-799 (2010).

102. X. Wang, B. Seed, A PCR primer bank for quantitative gene expression analysis. Nucleic Acids Res 31, e154 (2003)

103. ThermoFisher, Technical Resources, 10010 - PBS, pH 7.4.

104. I. I. I. C. M. Ofner, W. A. Bubnis, Pharmaceutical Research 13, 1821-1827 (1996).

105. S. Kasapis, I. M. Al-Marhoobi, J. R. Mitchell, Molecular weight effects on the glass transition of gelatin/cosolute mixtures. Biopolymers 70, 169-185 (2003).

106. T. J. Berg JM, Stryer L., in Biochemistry. 5th edition. ( W H Freeman, New York, 2002).

107. I. Pezron, M. Djabourov, J. Leblond, Conformation of gelatin chains in aqueous solutions: 1 . A light and small-angle neutron scattering study. Polymer 32, 3201-3210 (1991).

108. A. Gupta, B. Mohanty, H. B. Bohidar, Flory temperature and upper critical solution temperature of gelatin solutions. Biomacromolecules 6, 1623-1627 (2005). 


\section{Acknowledgments:}

The authors would like to acknowledge Dr. Barbara Pilas of the Roy J. Carver Biotechnology Center (Flow Cytometry Facility, UIUC) as well as Dr. Bhushan Mahadik and Dr. Ji-Sun Choi (ChBE, UIUC) for assistance with bone marrow cell isolation and flow cytometry. Preliminary Young's modulus testing and mRNA extraction was aided by undergraduate researchers Kirsten Schroeder and Gabrielle Wolter respectively. Research reported in this publication was supported by the National Institute of Diabetes and Digestive and Kidney Diseases of the National Institutes of Health under Award Numbers R01 DK099528 (B.A.C.H) and F31 DK1 17514 (A.E.G.), as well as by the National Institute of Biomedical Imaging and Bioengineering of the National Institutes of Health under Award Numbers R21 EB018481 (B.A.C.H.) and T32 EB019944 (A.E.G.). The content is solely the responsibility of the authors and does not necessarily represent the official views of the NIH. Amino Acid Analysis was performed at the Molecular Structure Facility in the UC Davis Genome Center at the University of California, Davis. Material characterization was carried out in part in the Frederick Seitz Materials Research Laboratory Central Research Facilities, University of Illinois. The authors are also grateful for additional funding provided by the Department of Chemical \& Biomolecular Engineering and the Institute for Genomic Biology at the University of Illinois at Urbana-Champaign. 


\section{Supplementary Materials:}
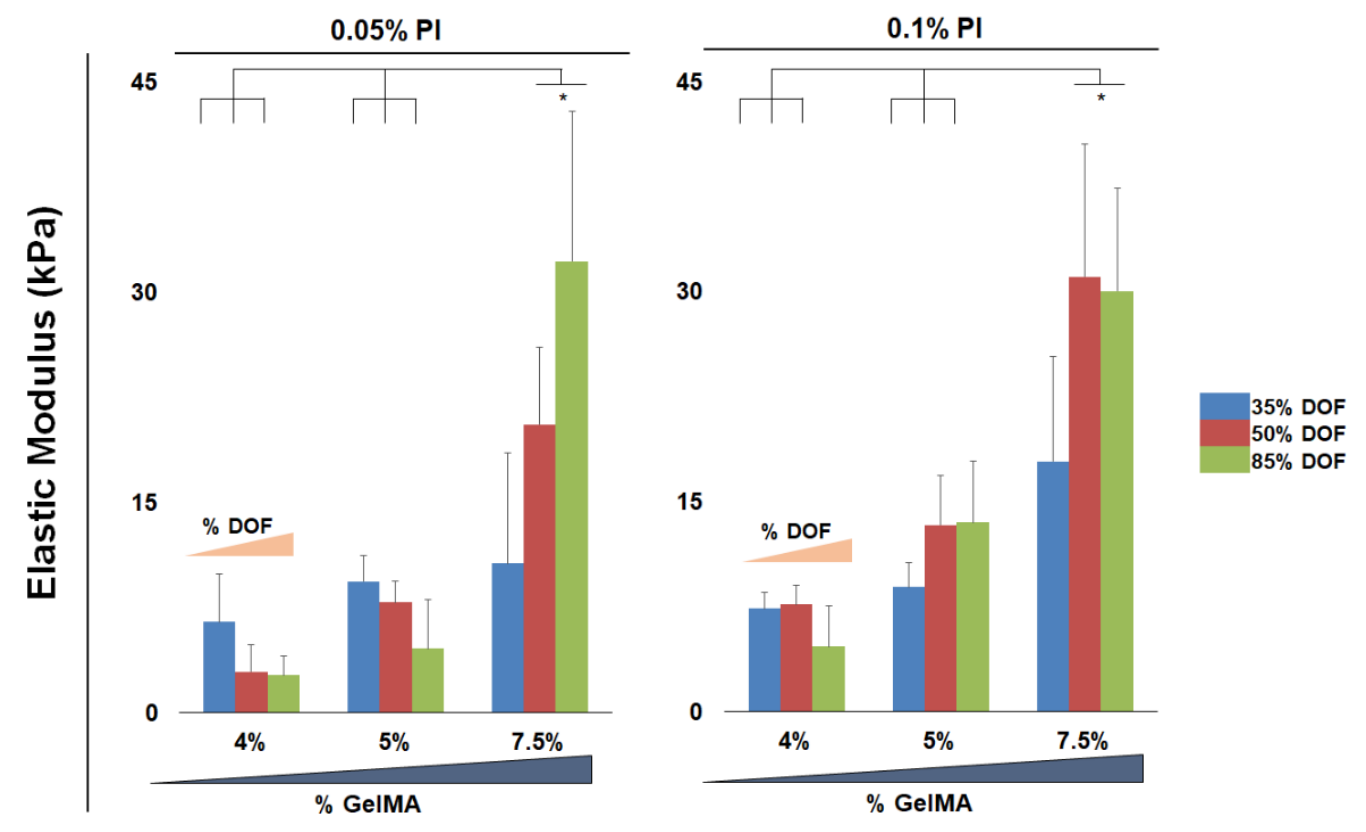

Supplemental Figure 1. Compressive mechanical analysis of the complete library of hydrogels.

The degree of methacrylamide-functionalization was altered from $35,50,85 \%$, and the content of GelMA was increased from 4, 5, 7.5\%. * marks significantly different at the $p<0.05$ level. $(\mathbf{n}=\mathbf{6})$. A) Elastic modulus of hydrogels prepared with $0.05 \%$ photoinitiator. B) Elastic modulus of hydrogels prepared with $0.1 \%$ photoinitiator. 

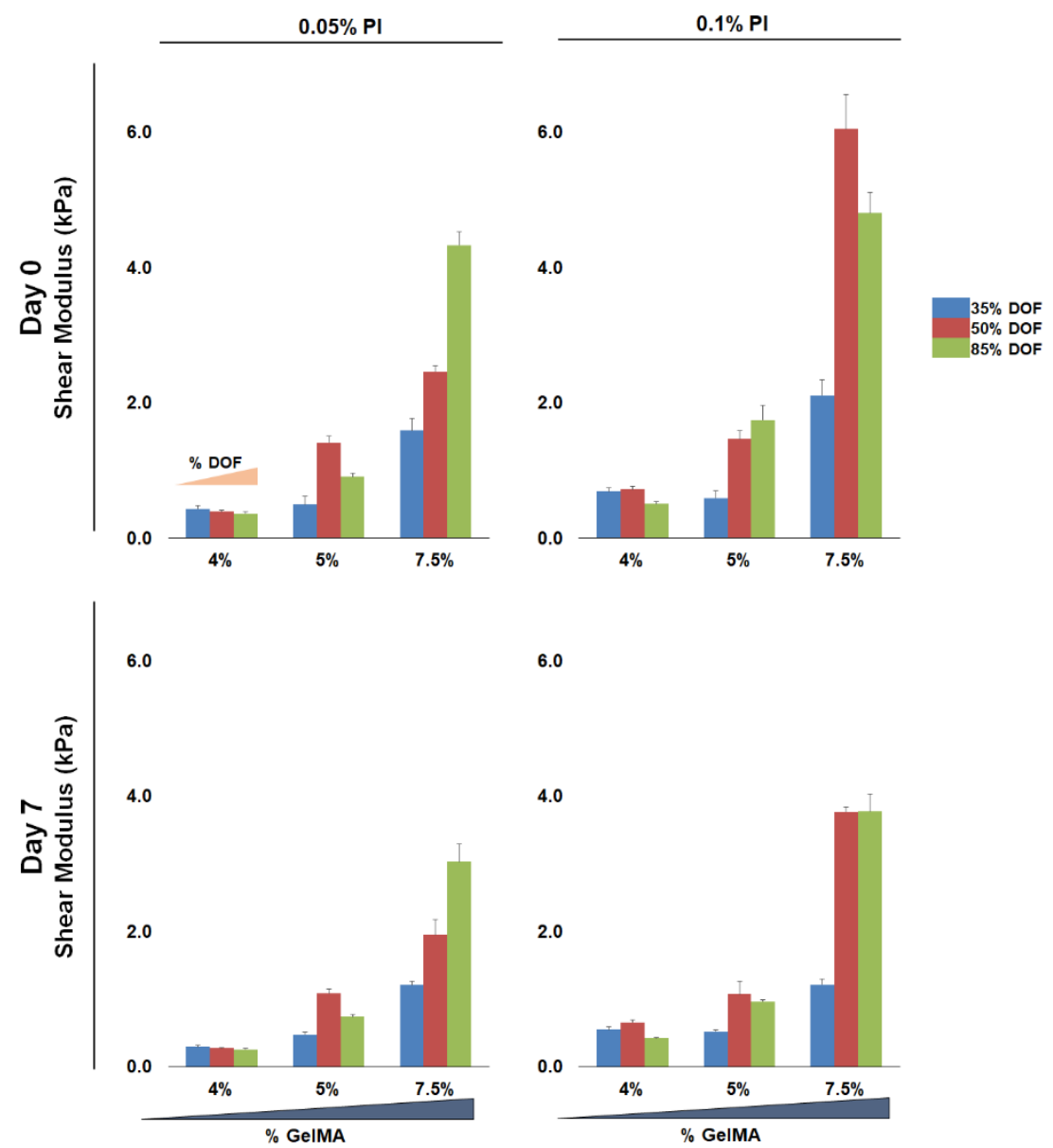

Supplemental Figure 2. Shear modulus characterization of the complete library of hydrogels.

The degree of methacrylamide-functionalization was altered from $35,50,85 \%$, and the content of GelMA was increased from 4, 5, 7.5\%. (n=6). A) Shear modulus of hydrogels prepared with $0.05 \%$ photoinitiator, measured at Day 0 after hydration in PBS. B) Shear modulus of hydrogels prepared with $0.1 \%$ photoinitiator, measured at Day 0 after hydration in PBS. C) Shear modulus of hydrogels prepared with $0.05 \%$ photoinitiator, measured at Day 7. D) Shear modulus of hydrogels prepared with $0.1 \%$ photoinitiator, measured at Day 7. 

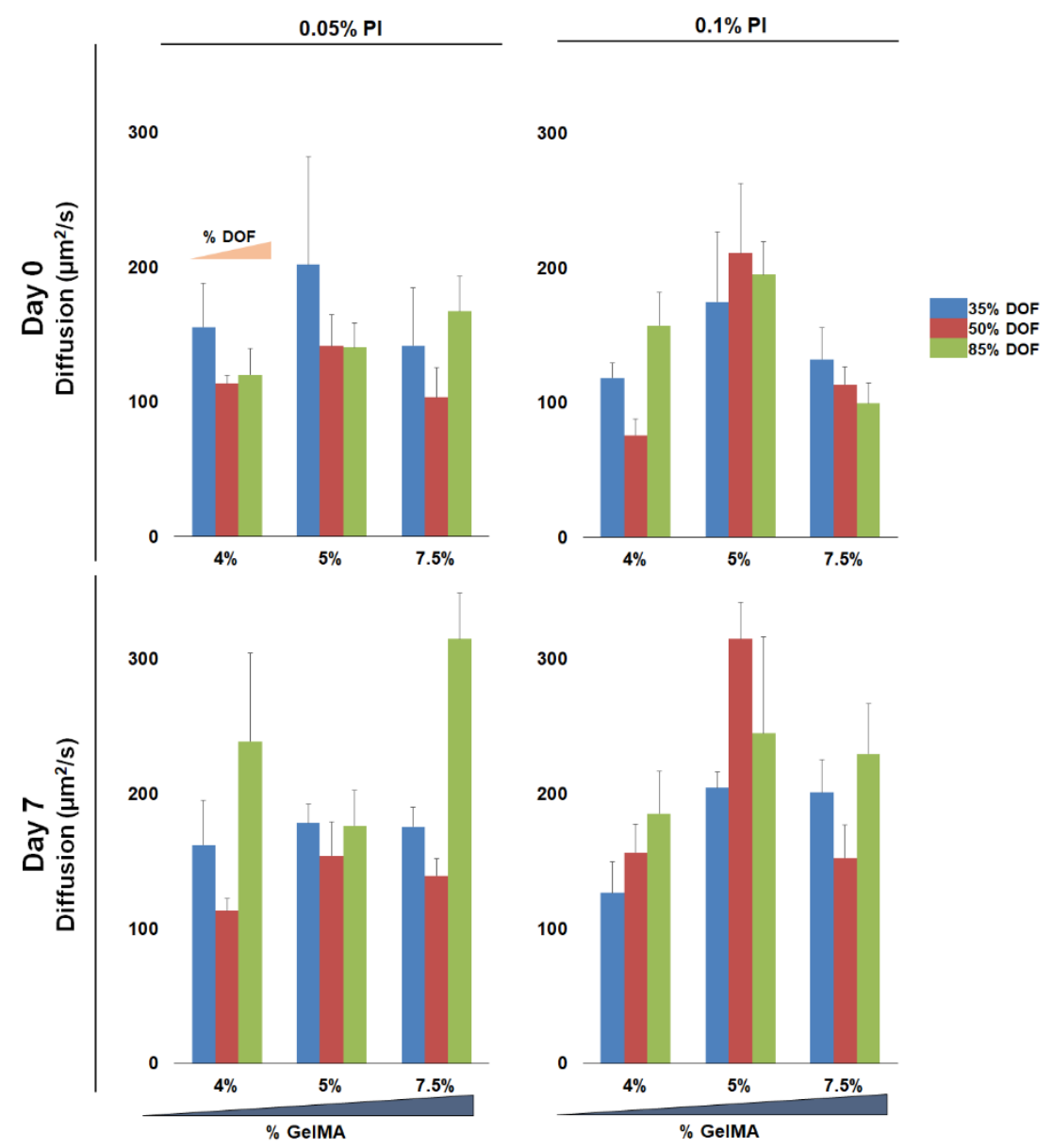

Supplemental Figure 3. The diffusion coefficient of water in each hydrogel was measured for the complete library of hydrogels. The degree of methacrylamide-functionalization was altered from 35, 50, 85\%, and the content of GelMA was increased from 4, 5, 7.5\%. (n=6). A) Diffusion coefficient of water in hydrogels prepared with $0.05 \%$ photoinitiator, measured at Day 0 after hydration in PBS. B) Diffusion coefficient of water in hydrogels prepared with $0.1 \%$ photoinitiator, measured at Day 0 after hydration in PBS. C) Diffusion coefficient of water in hydrogels prepared with $0.05 \%$ photoinitiator, measured at Day 7. D) Diffusion coefficient of water in hydrogels prepared with $0.1 \%$ photoinitiator, measured at Day 7 . 


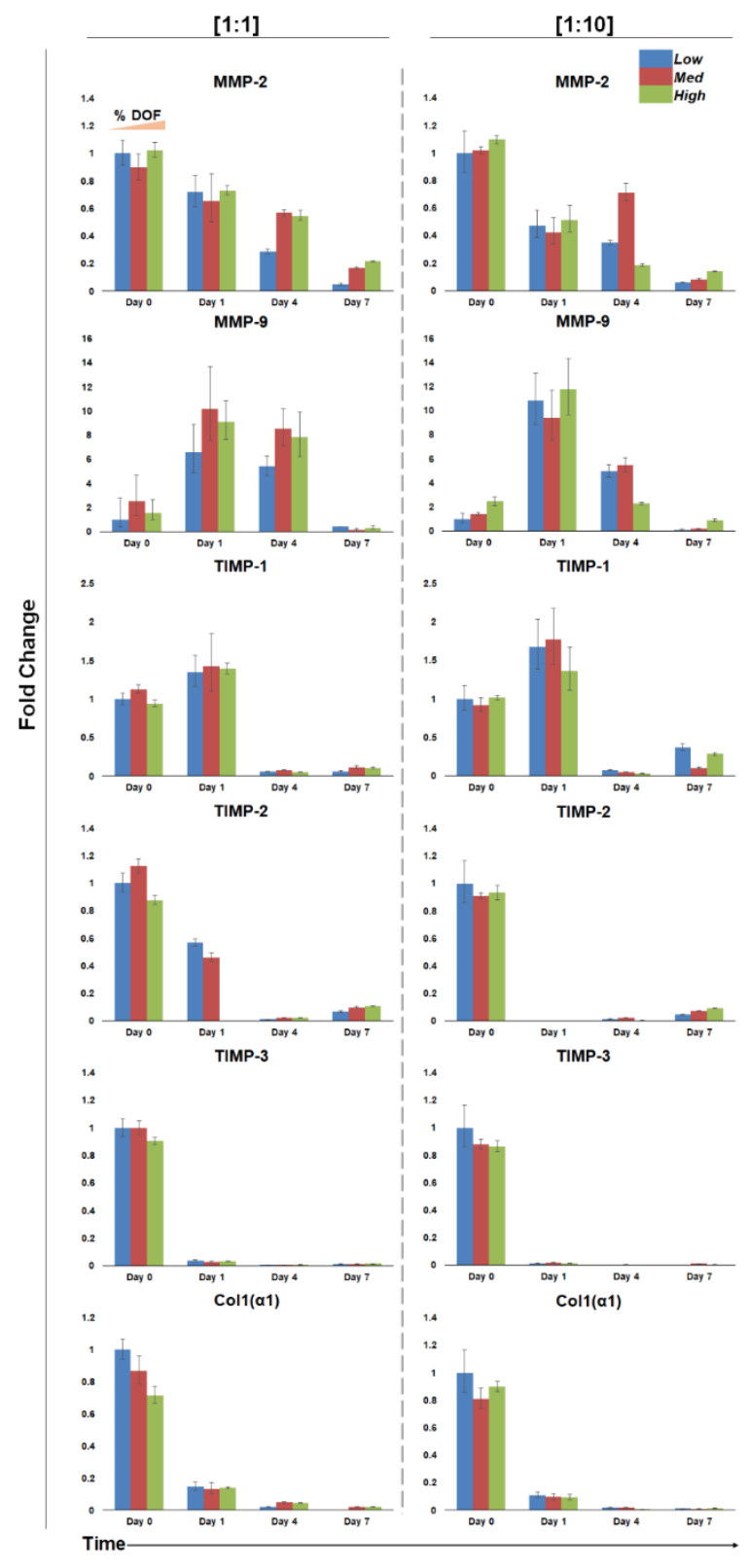

Supplemental Figure 4. Relative expression of matrix-associated genes in Low, Med, and High hydrogels seeded with $1 \times 10^{5}(1: 1)$ and $1 \times 10^{6} \mathrm{MSCs} / \mathrm{mL}$ (1:10). GAPDH was the housekeeping gene, and expression was normalized to Day 0 of the low hydrogel condition. $(\mathbf{n = 3 - 6})$. A) Relative gene expression of hydrogels seeded with $1 \times 10^{5} \mathrm{MSCs} / \mathrm{mL}(1: 1)$ B) Relative gene expression of hydrogels seeded with $1 \times 10^{6} \mathrm{MSCs} / \mathrm{mL}(1: 10)$. 


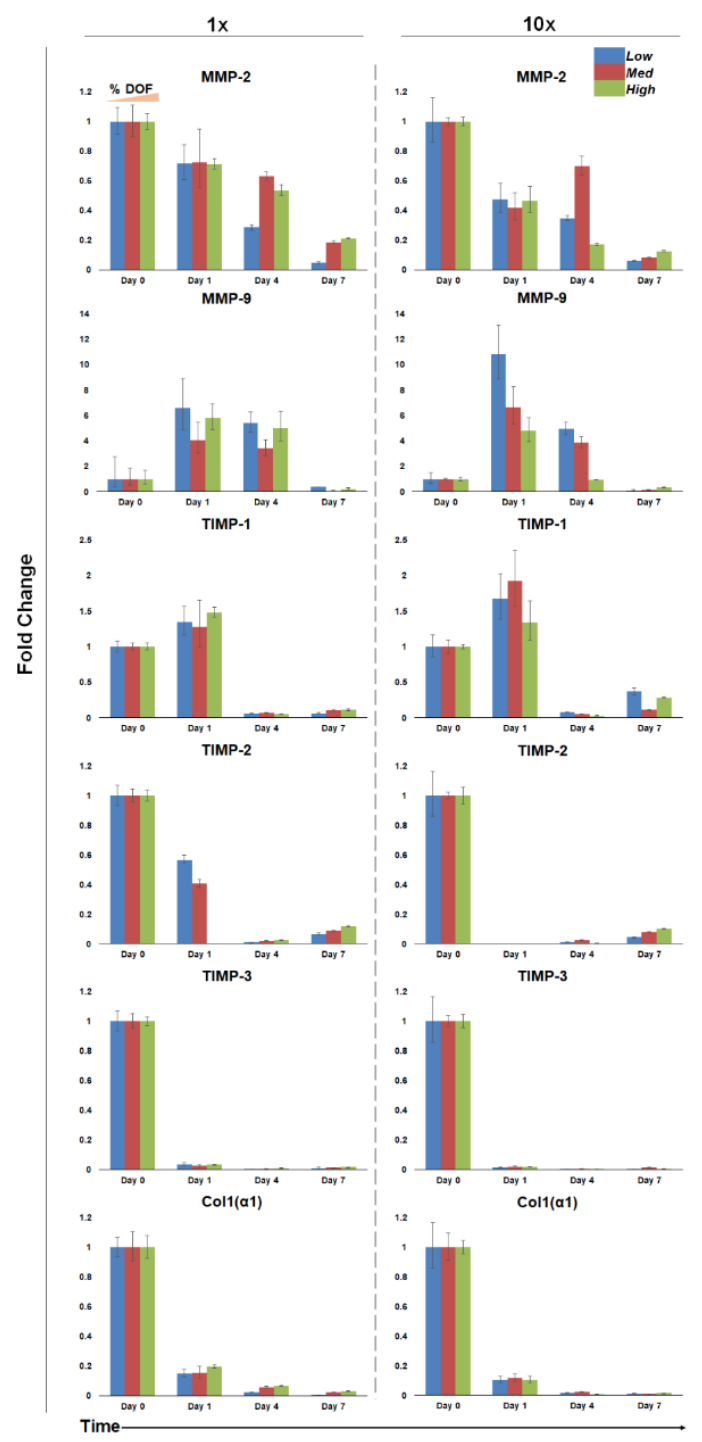

Supplemental Figure 5. Relative expression of matrix-associated genes in Low, Med, and High hydrogels seeded with $1 \times 10^{5}(1: 1)$ and $1 \times 10^{6} \mathrm{MSCs} / \mathrm{mL}$ (1:10). GAPDH was the housekeeping gene, and expression was normalized to Day 0 of the specific hydrogel and seeding condition. (n=3-6). A) Relative gene expression of hydrogels seeded with $1 \times 10^{5} \mathrm{MSCs} / \mathrm{mL}(1: 1)$. B) Relative gene expression of hydrogels seeded with $1 \times 10^{6} \mathrm{MSCs} / \mathrm{mL}(1: 10)$. 

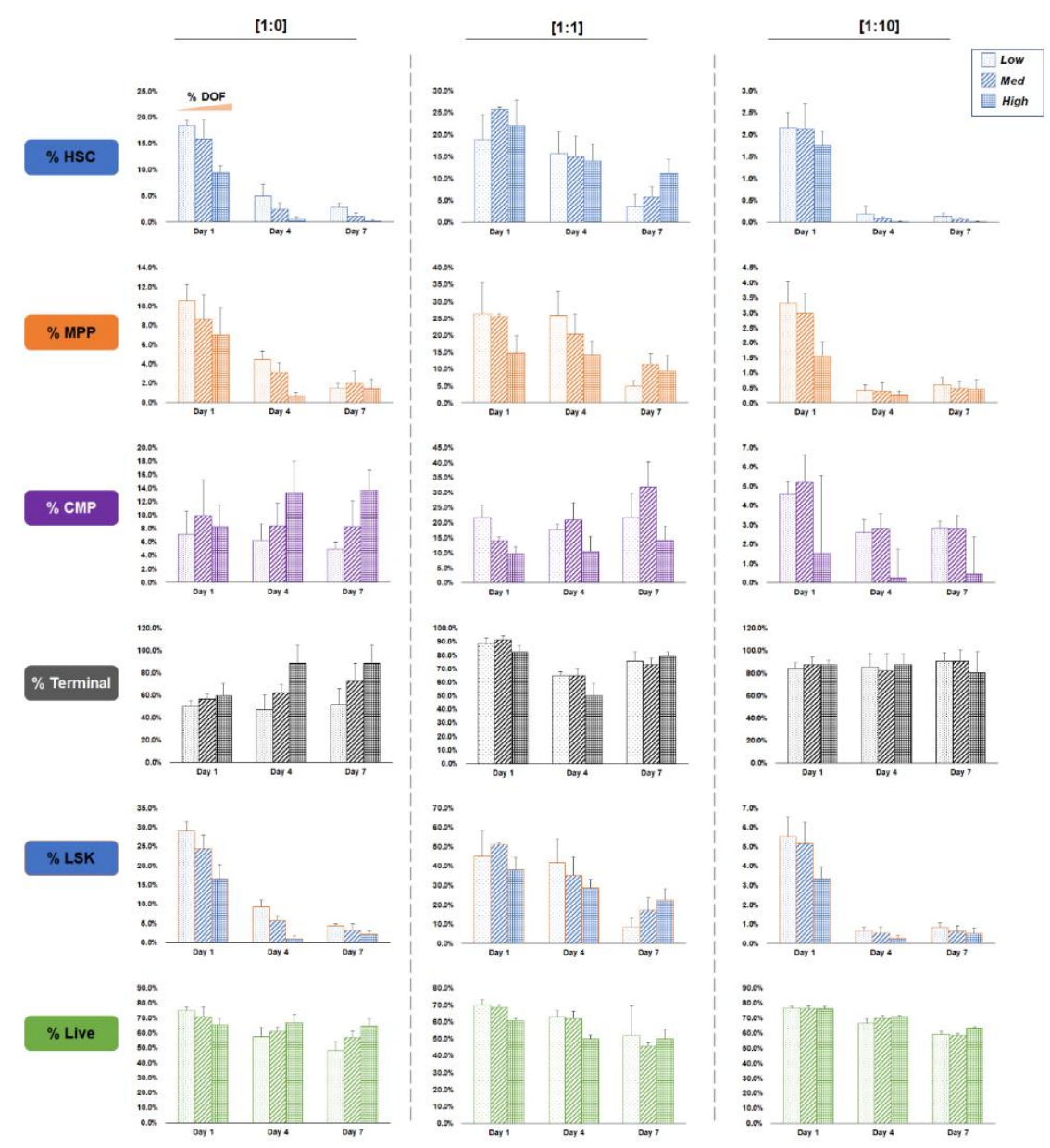

Supplemental Figure 6. Hematopoietic cell differentiation patterns within Low, Med, and High hydrogels with $0,1 \times 10^{5}$, and $1 \times 10^{6} \mathrm{MSCs} / \mathrm{mL}(1: 0,1: 1$, and 1:10 respectively). Each cell type is expressed as a percentage of the overall hematopoietic cell population. $(\mathbf{n = 6})$. A) Hematopoietic stem cell population, comprised of long-term and short-term HSCs. B) Multipotent progenitor cells. C) Common myeloid progenitor cells. D) Terminal cell population, comprised of all nonLSK hematopoietic cells. E) LSK cells comprised of LT-HSC, ST-HSCs, and MPPs. F) The live population of cells expressed as a percentage of live cells over the total population of all cells. 
A

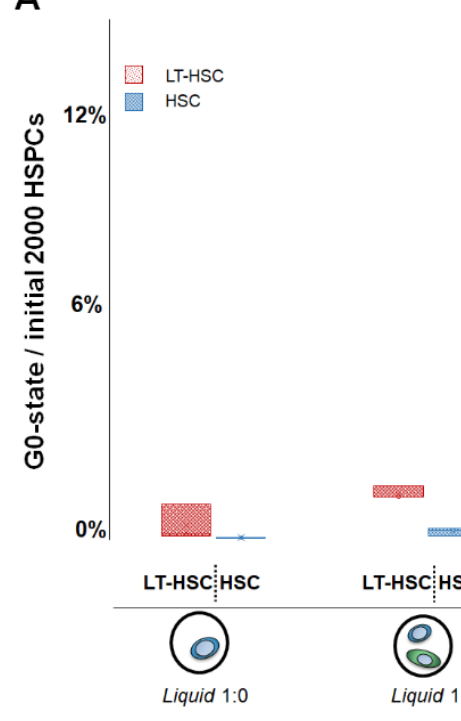

B

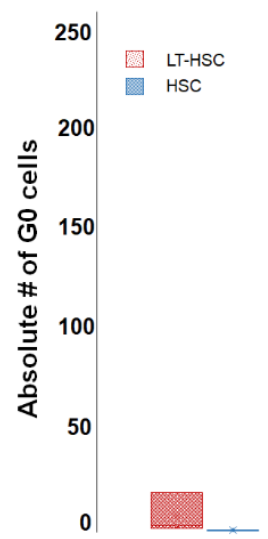

250

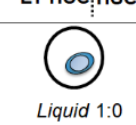

C

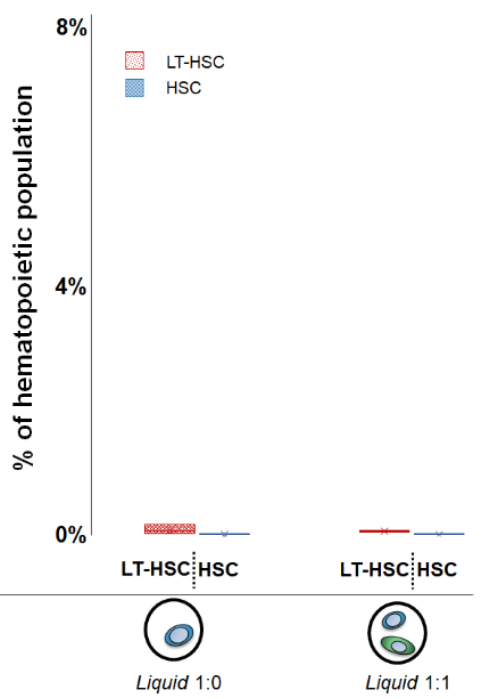

Supplemental Figure 7. Number and fraction of quiescent LT-HSCs and HSCs in liquid culture containing either $1 \times 10^{5} \mathrm{HSPCs} / \mathrm{mL}$ alone (1:0), or a mixture of $1 \times 10^{5} \mathrm{HSPCs} / \mathrm{mL}$ and $1 \times 10^{5}$ MSCs/mL (1:1). (n=3). A) Number of quiescent LT-HSC or HSC, shown as a percentage of the initial 2000 HSPCs seeded. B) The absolute number of quiescent (G0) LT-HSC and HSCs within each sample (both populations are highest in the High variant at 1:1 seeding). C) Percentage of quiescent cells calculated vs. the total number of hematopoietic lineage positive cells after 7 days in culture (including hematopoietic progeny that arise during the culture period). 


\section{Supplemental Table 1.}

Forward and reverse primer sequences for matrix associated genes. Sequences were made using

NCBI Primer-blast or taken from literature.

\begin{tabular}{|l|l|l|l|l|}
\hline Name & Accession \# & Forward & Reverse & Citation \\
\hline Col1 $(\alpha 1)$ & NM_007742.4 & CGACCTCAAGATGTGCCACT & GACGGCTGAGTAGGGAACAC & $(99)$ \\
\hline MMP-2 & NM_008610.3 & CCTGACCTGGACCCTGAAAC & TCCCAGCGTCCAAAGTTGAT & $(99)$ \\
\hline MMP-9 & $\begin{array}{l}\text { NM_013599.4 } \\
\text { PB\#: } 31560795 c 2\end{array}$ & GGACCCGAAGCGGACATTG & CGTCGTCGAAATGGGCATCT & $($ 100-102) \\
\hline TIMP-1 & NM_001044384.1 & TGGCATCTGGCATCCTCTTG & GGTCTCGTTGATTTCTGGGGA & $(99)$ \\
\hline TIMP-2 & NM_011594.3 & TATCTACACGGCCCCCTCTT & TCCCAGGGCACAATGAAGTC & $($ 99) \\
\hline TIMP-3 & NM_011595.2 & TCTCTGGACCGACATGCTCT & GCGTTGCTGATGCTCTTGTC & $($ 99) \\
\hline GAPDH & NM_001289726.1 & TGTGTCCGTCGTGGATCTGA & CCTGCTTCACCACCTTCTTGA & (33) \\
\hline
\end{tabular}




\section{Supplemental Table 2.}

Parameters for hydrogel mesh calculations. Values with references were either taken directly or interpolated from literature.

\begin{tabular}{|l|c|c|c|c|c|}
\hline Name & Variables & Low & Med & High & Citation \\
\hline Relaxed mass swelling ratio & $Q_{m r}$ & 32.46 & 22.01 & 14.80 & \\
\hline Equilibrium mass swelling ratio & $Q_{m}$ & 26.39 & 19.59 & 11.97 & \\
\hline polymer density $\left[\mathrm{g} / \mathrm{cm}^{3}\right.$ ] & $\rho_{p}$ & 1.35 & 1.35 & 1.35 & $(74,75)$ \\
\hline Solvent density $\left[\mathrm{g} / \mathrm{cm}^{3}\right]$ & $\rho_{s}$ & 1.014 & 1.014 & 1.014 & $(73,103)$ \\
\hline Relaxed volumetric swelling & $Q_{v r}$ & 42.87 & 28.96 & 19.37 & \\
\hline Equilibrium volumetric swelling & $Q_{v}$ & 34.79 & 25.75 & 15.60 & \\
\hline Relaxed polymer volume fraction & $v_{2 r}$ & 0.0233 & 0.0345 & 0.0516 & \\
\hline Equilibrium polymer volume fraction & $v_{2 s}$ & 0.0287 & 0.03889 & 0.0641 & \\
\hline Polymer-solvent interaction & $\chi$ & 0.497 & 0.497 & 0.497 & $(104)$ \\
\hline Molar volume of water [mL/mol] & $V_{1}$ & 18.01 & 18.01 & 18.01 & \\
\hline Specific volume of polymer [mL/g] & $\bar{v}$ & 0.7407 & 0.7407 & 0.7407 & \\
\hline Number average molecular weight before crosslink & $M_{n}$ & 63565.35 & 63565.35 & 63565.35 & $(105)$ \\
\hline Molecular weight between crosslinks [g/mol] & $M_{c}$ & 13825.63 & 10759.50 & 4654.85 & \\
\hline Mean squared distance & $M_{c}$ & 49316.40 & 38379.42 & 16603.97 & \\
\hline Bond length $(\dot{A})$ & $\left.l^{2}\right) C_{r}$ & & & & $(106)$ \\
\hline Persistence length $(\dot{A})$ & $l$ & 4.28 & 4.28 & 4.28 & $(107$, \\
\hline Molecular weight of the repeat unit & $l_{p}$ & 20 & 20 & 20 & $108)$ \\
\hline Flory characteristic ratio & $M_{r}$ & 91.19 & 91.19 & 91.19 & \\
\hline Mesh size (nanometers) & $C_{n}$ & 8.8785 & 8.8785 & 8.8785 & \\
\hline
\end{tabular}

